\title{
A general construction of conformal field theories from scalar anti-de Sitter quantum field theories
}

\author{
Marco Bertola ${ }^{\mathrm{a}, *}$, Jacques Bros ${ }^{\mathrm{b}}$, Ugo Moschella ${ }^{\mathrm{b}, \mathrm{c}}$, Richard Schaeffer ${ }^{\mathrm{b}}$ \\ a CRM, Universite' de Montreal, Montreal, PQ, H3C 3J7, Canada \\ b Service de Physique Théorique, C.E. Saclay, 91191 Gif-sur-Yvette, France \\ c Dipartimento di Scienze Matematiche Fisiche e Chimiche, Università dell'Insubria, 22100 Como and INFN \\ sezione di Milano, Milano, Italy
}

Received 24 August 1999; revised 22 June 2000; accepted 17 July 2000

\begin{abstract}
We provide a new general setting for scalar interacting fields on the covering of a $(d+1)$ dimensional AdS spacetime. The formalism is used at first to construct a one-parameter family of field theories, each living on a corresponding spacetime submanifold of AdS, which is a cylinder $\mathbb{R} \times$ $\mathbb{S}_{d-1}$. We then introduce a limiting procedure which directly produces Lüscher-Mack CFT's on the covering of the AdS asymptotic cone. Our generalized AdS $\rightarrow$ CFT construction is nonperturbative, and is illustrated by a complete treatment of two-point functions, the case of Klein-Gordon fields appearing as particularly simple in our context.

We also show how the Minkowskian representation of these boundary CFT's can be directly generated by an alternative limiting procedure involving Minkowskian theories in horocyclic sections (nowadays called ( $d-1$ )-branes, 3 -branes for $A d S_{5}$ ). These theories are restrictions to the brane of the ambient AdS field theory considered. This provides a more general correspondence between the AdS field theory and a Poincare invariant QFT on the brane, satisfying all the Wightman axioms. The case of two-point functions is again studied in detail from this viewpoint as well as the CFT limit on the boundary. () 2000 Elsevier Science B.V. All rights reserved.
\end{abstract}

PACS: 11.10.Cd; 04.62.+v

Keywords: AdS quantum field theory; Conformal field theory

\section{Introduction}

The by-now famous Maldacena's AdS/CFT correspondence conjectures a duality between type IIB superstring theory on $A d S_{5} \times S_{5}$ and super Yang-Mills conformal

\footnotetext{
* Corresponding author.

E-mail address: bertola@crm.umontreal.ca (M. Bertola).
} 
quantum field theory (QFT) on the flat four-dimensional boundary of $A d S_{5}$ (times the five sphere $S_{5}$ ) [1].

Not only proving the conjecture in his original shape is far beyond today's possibilities (as one would need to solve both superstring and super Yang-Mills theories and then establish the duality), but also its very formulation is not trivial. As a matter of fact, the original seminal paper by Maldacena [1] did not spell out how the correspondence (or duality) should precisely work.

A way to make effective a weaker version of the conjecture, which relates the boundary conformal field theory (CFT) with the supergravity approximation to superstring theory has been subsequently formulated independently by Gubser, Klebanov and Polyakov in [2] and by Witten in [3]. This proposal prescribes how to compute the Green's functions of the boundary (super Yang-Mills) CFT in terms of the Euclidean classical supergravity action of an AdS field configuration which obeys to precise boundary conditions and it has been discussed in a large number of papers (for a review see [4]). Most of these works are making use of classical field theory methods, mainly in a Euclidean formulation allowing the use of Dirichlet's problem but also in a Lorentzian formulation in terms of modes of the Klein-Gordon equation [5].

The ideas of the AdS/CFT conjecture also suggest the existence of a rich and yet uninvestigated conceptual content at the level of quantum fields and therefore it is natural that many works in this domain have also made use of the already existing (and more than two decades old) results QFT on the AdS spacetime. In this connection, one must quote the pioneering approach of [6], whose main concern was to specify boundary conditions such that the difficulties arising by the lack of global hyperbolicity of the underlying AdS manifold could be circumvented and the resulting QFT be well defined. Another, earlier, approach was also given on the basis of group-theoretical methods [7] following ideas that can be traced back to Dirac [8]. Both of these approaches have influenced the research on the AdS/CFT subject. However, their applicability is more or less limited to free AdS QFT's even if they can produce useful ingredients for perturbative calculations (mainly tree-level calculations [11,12], but also involving in special cases nontrivial bubbles of four-point functions [13]).

To go beyond, it may be useful to consider the AdS/CFT debate on a more general basis ${ }^{1}$ in which both AdS quantum fields and boundary CFT's would be treated from the viewpoint of the structural properties of their $n$-point correlation functions. This approach could also have a practical value for a general perturbative renormalization theory.

It is precisely at this level that this paper wants to address the issue, by studying the relationship that exists between scalar AdS QFT's and CFT's living on the boundary of the AdS manifold. To this end, we introduce a (minimal) set of field-theoretical requirements in order to give a well-posed framework for the study of AdS QFT's. Then we spell out a condition on AdS fields that allows one to associate a well-defined CFT on the boundary

\footnotetext{
${ }^{1}$ In this spirit such a setting has been proposed [9] in the general framework of algebras of local observables (or "local quantum physics" in the sense of Haag [10]).
} 
of the AdS spacetime with any given (interacting, nonperturbative) scalar field theory in the AdS bulk that satisfies it. We call this property asymptotic dimensionality condition.

The result of our study could therefore be summarized by the symbolic writing AdS $\rightarrow$ CFT (as opposed to AdS/CFT), since, given any scalar QFT satisfying our set of axioms and the asymptotic dimensionality condition, we are guaranteed that there exist a fully acceptable boundary CFT and we know how to construct it.

We will not address in this paper the question of the applicability of this general correspondence to the original Maldacena's AdS/CFT conjecture. This would require a more elaborate scheme taking into account also supersymmetry.

It might be that the original AdS/CFT correspondence (in its field theoretical formulations by Gubser, Klebanov and Polyakov and by Witten) is a subset of our AdS $\rightarrow$ CFT construction. This possibility is corroborated by the fact that, with our method, we can reproduce in a really elementary way the well-known relation between AdS masses and boundary conformal weights [3] and the various two-point functions. The question however cannot be answered without first showing that there exists a supergravity theory satisfying a set of axioms similar to those that we spell out for scalar theories, and it is well-known that supergravity is an effective theory possibly non-existent as an acceptable QFT. However, it is also well known that a seemingly innocent question like that, for the much better understood quantum electrodynamics (and, even simpler, for one scalar selfinteracting field in four spacetime dimensions), has stayed unanswered for seventy-five years up to date.

Also, we do not dwell on the converse relation, ${ }^{2}$ which is also needed to establish a duality (rather than a projection). In this respect, our concern is certainly simpler but, at the same time, it promises a more general outcome: it always gives a boundary CFT given a (scalar) AdS QFT satisfying our hypotheses.

Let us give now a few more technical details and the plan of the paper. Our approach is based on a basic work by Lüscher and Mack [14], where the concept of global conformal invariance in Minkowskian QFT has been associated in a deep and fruitful way with the general framework of QFT on the covering of a quadratic cone with signature $(+,+,-, \cdots,-)$ (in one dimension more). Since such a cone is precisely the asymptotic cone of the AdS quadric, it seems quite appropriate to try to formulate our axiomatic AdS $\rightarrow$ CFT relation in a way which exhibits as clearly as possible the connection between Lüscher's and Mack's QFT framework on the cone with a similar QFT framework on the AdS quadric. This is the first purpose of the present work.

In Section 2 we introduce such a general framework for the study of quantum fields on a $(d+1)$-dimensional AdS spacetime $A d S_{d+1}$ (or more precisely on its covering) in the spirit of [15]. Then, in Section 3, we present a direct and simple method for obtaining correlation functions of conformal fields on the boundary, method which does not rely on the use of any field equation.

\footnotetext{
${ }^{2}$ The existence of such a converse relation has been established in [9] CFT $\rightarrow$ AdS at a more global level, namely for algebras of local observables.
} 
General interacting QFT's on the (covering of the) AdS spacetime are assumed to satisfy a set of basic properties such as microcausality, AdS covariance and a certain energy spectrum condition here introduced in terms of appropriate analyticity properties of the $n$-point functions. Together with these general properties, it is also crucial to require a certain type of asymptotic behavior for the $n$-point functions in terms of an asymptotic dimensionality condition. The possibility to use such type of asymptotic condition has been mentioned in a perturbative context in [16]. We then introduce a limiting procedure which directly produces CFT's (in the sense of [14]) on the covering of the asymptotic cone of AdS spacetime; the conformal covariance of the corresponding Minkowskian (interacting) field theory on the boundary is then proved without making use of any field equation.

This difference from the original largely followed proposals of [2] and [3] is worth noticing: rather than solving explicitly for a field that is defined by suitable boundary conditions on the edge - which can be done in a tractable way only for free fields - we define a limiting procedure that can be applied directly to the AdS correlation functions. This is much simpler already for free fields, but our treatment also shows how to deal with interacting fields $A d S_{d+1}$ and we gain a general nonperturbative foundation for a scalar AdS $\rightarrow$ CFT construction formulated in terms of the $n$-point correlation functions of such fields and treated from a model-independent point of view.

In Section 4 we provide a complete treatment of two-point correlation functions. By applying the general setting of Section 2 we are able to exhibit strong analyticity properties of AdS two-point functions [17], which are closely similar to those enjoyed by two-point functions in flat spacetime or in de Sitter spacetime [18,19]. In the case of Klein-Gordon fields, these analyticity properties fix completely their form to be necessarily appropriate second-kind Legendre functions, as obtained (for the four-dimensional case) in the grouptheoretical approach of [7]; the CFT limits of such two-point functions are then directly computable in full consistency with the given general formulation of the AdS $\rightarrow$ CFT correspondence.

We also provide an alternative construction based on the Poincaré or horocyclic coordinate system for $A d S_{d+1}$. This way of looking to the AdS spacetime as a warped manifold with Poincaré sections (today called $(d-1)$-branes) has recently gained an enormous interest in a phenomenological and cosmological context [20].

We show that by restriction, AdS correlation functions satisfying our general properties of Section 2 define acceptable two-point Minkowskian QFT correlation functions on the branes (corresponding to flat $d$-dimensional spacetime sections of the AdS manifold).

In particular, in Section 5 we establish a spectral decomposition for the AdS KleinGordon fields naturally associated to the Poincare foliation of the AdS quadric. In this scheme the well-known ambiguity for low mass theories [21] is seen to be linked to the lack of essential self-adjointness of the Bessel's differential operator [22] which naturally arises in the Poincare coordinates.

In the last part of our paper, we introduce a more general possible use of the Poincaré foliation by showing how general Minkowskian interacting QFT's can be produced by taking the restrictions of general AdS QFT's to the branes. It is worthwhile to stress that this is a nontrivial step. Indeed it is well known that, as a consequence of Heisenberg's 
indetermination principle, in general a quantum field cannot be restricted to lowerdimensional submanifolds of the spacetime where the field live. It is a bonus of our axioms which allows this restriction of an AdS quantum field to a Poincaré or cylindrical brane. Moreover, by sending these leaves to infinity we exhibit a limiting procedure for the Minkowskian QFT's in the leaves which provides an alternative presentation of the previously defined AdS $\rightarrow$ CFT construction. The interest of this presentation is that it is entirely expressed in terms of Minkowskian theories satisfying the Wightman axioms, and can be of interest for the multidimensional approach to phenomenology and cosmology [20].

\section{General QFT in AdS spacetime}

\subsection{Notations and geometry}

We consider the vector space $\mathbb{R}^{d+2}$ equipped with the following pseudo-scalar product:

$$
X \cdot X^{\prime}=X^{0} X^{\prime 0}-X^{1} X^{\prime 1}-\cdots-X^{d} X^{\prime d}+X^{d+1} X^{\prime d+1} \text {. }
$$

The $(d+1)$-dimensional AdS universe can then be identified with the quadric

$$
A d S_{d+1}=\left\{X \in \mathbb{R}^{d+2}, X^{2}=R^{2}\right\},
$$

where $X^{2}=X \cdot X$, endowed with the induced metric

$$
\mathrm{d} s_{A d S}^{2}=\left.\left(\mathrm{d} X^{0^{2}}-\mathrm{d} X^{1^{2}}-\cdots+\mathrm{d} X^{d+1^{2}}\right)\right|_{A d S_{d+1}} .
$$

The AdS relativity group is $G=S O_{0}(2, d)$, that is the component connected to the identity of the pseudo-orthogonal group $O(2, d)$. Two events $X, X^{\prime}$ of $A d S_{d+1}$ are space-like separated if $\left(X-X^{\prime}\right)^{2}<0$, i.e., if $X \cdot X^{\prime}>R^{2}$.

We will also consider the complexification of $A d S_{d+1}$ :

$$
A d S_{d+1}^{(c)}=\left\{Z=X+i Y \in \mathbb{C}^{d+2}, Z^{2}=R^{2}\right\} .
$$

In other terms, $Z=X+i Y$ belongs to $A d S_{d+1}^{(c)}$ if and only if $X^{2}-Y^{2}=R^{2}$ and $X \cdot Y=0$. In the following we will put for notational simplicity $R=1$.

We shall make use of two parametrizations for the AdS manifold.

The "covering parametrization" $X=X[r, \tau, \mathrm{e}]$ : it is obtained by intersecting $A d S_{d+1}$ with the cylinders with equation $\left\{X^{0^{2}}+X^{d+1^{2}}=r^{2}+1\right\}$, and is given by

$$
\left\{\begin{array}{l}
X^{0}=\sqrt{r^{2}+1} \sin \tau, \\
X^{i}=r \mathrm{e}^{i}, \quad i=1, \ldots, d, \\
X^{d+1}=\sqrt{r^{2}+1} \cos \tau,
\end{array}\right.
$$

with $\mathrm{e}^{2} \equiv \mathrm{e}^{1^{2}}+\cdots+\mathrm{e}^{d^{2}}=1$ and $r \geqslant 0$. For each fixed value of $r$, the corresponding "slice"

$$
C_{r}=A d S_{d+1} \cap\left\{X^{0^{2}}+X^{d+1^{2}}=r^{2}+1\right\}
$$


of $A d S_{d+1}$ is a manifold $\mathbb{S}_{1} \times \mathbb{S}_{d-1}$. The complexified space $A d S_{d+1}^{(c)}$ is obtained by giving arbitrary complex values to $r, \tau$ and to the coordinates $\mathrm{e}=\left(\mathrm{e}^{i}\right)$ on the unit $(d-1)$-sphere.

The parametrization (5) allows one to introduce relevant coverings of $A d S_{d+1}$ and $A d S_{d+1}^{(c)}$ by unfolding the $2 \pi$-periodic coordinate $\tau$ (respectively, $\operatorname{Re} \tau$ ), interpreted as a time-parameter: these coverings are denoted, respectively, by $\widehat{\operatorname{AdS}}_{d+1}$ and $\widehat{\operatorname{AdS}}_{d+1}^{(c)}$. A privileged "fundamental sheet" is defined on these coverings by imposing the condition $-\pi<\tau<\pi$ (respectively, $-\pi<\operatorname{Re} \tau<\pi$ ). This procedure also associates with each manifold $C_{r}$ its covering $\widehat{C}_{r}$ which is a cylinder $\mathbb{R}_{\tau} \times \mathbb{S}_{d-1 \mathrm{e}}$. We will use the symbols $X$, $Z, \ldots$, also to denote points of the coverings.

Similarly one introduces a covering $\widehat{G}$ of the group $G$ by taking in $G$ the universal covering of the rotation subgroup in the $(0, d+1)$-plane. By transitivity, $A d S_{d+1}$ and $\widehat{A d S}_{d+1}$ are, respectively, generated by the action of $G$ and $\widehat{G}$ on the base point $B=$ $(0, \ldots, 0,1)$.

The physical reason which motivates the introduction of the covering $\widehat{A d S}_{d+1}$, that is the requirement of nonexistence of closed time-loops, also leads us to specify the notion of spacelike separation in $\widehat{A d S}_{d+1}$ as follows: let $X, X^{\prime} \in \widehat{A d S}_{d+1}$ and let $g$ an element of $\widehat{G}$ such that $X^{\prime}=g B$; define $X_{g}=g^{-1} X . X$ and $X^{\prime}$ are spacelike separated if $X_{g}$ is in the fundamental sheet of $\widehat{A d S}_{d+1}$ and $\left(X-X^{\prime}\right)^{2} \equiv\left(g^{-1} X-g^{-1} X^{\prime}\right)^{2}<0$. This implies that $X_{g}=X_{g}[r, \tau, \mathrm{e}]$ with $-\pi<\tau<\pi$ and $\sqrt{r^{2}+1} \cos \tau>1$.

It is also interesting to note that on each manifold $C_{r}$ the condition of spacelike separation between two points $X=X[r, \tau, \mathrm{e}]$ and $X^{\prime}=X^{\prime}\left[r, \tau^{\prime}, \mathrm{e}^{\prime}\right]$ reads (in view of (5)):

$$
\left(X-X^{\prime}\right)^{2}=2\left(r^{2}+1\right)\left(1-\cos \left(\tau-\tau^{\prime}\right)\right)-r^{2}\left(\mathrm{e}-\mathrm{e}^{\prime}\right)^{2}<0,
$$

and that the corresponding covering manifold $\widehat{C}_{r}$ therefore admits a global causal ordering which is specified as follows:

$$
(\tau, \mathrm{e})>\left(\tau^{\prime}, \mathrm{e}^{\prime}\right), \quad \text { iff } \tau-\tau^{\prime}>2 \operatorname{Arcsin}\left(\frac{\left(\mathrm{e}-\mathrm{e}^{\prime}\right)^{2}}{4} \frac{r^{2}}{r^{2}+1}\right)^{1 / 2} .
$$

The "horocyclic parametrization" $X=X(v, x)$ : it only covers the part $\Pi$ of the AdS manifold which belongs to the half-space $\left\{X^{d}+X^{d+1}>0\right\}$ of the ambient space and is obtained by intersecting $A d S_{d+1}$ with the hyperplanes $\left\{X^{d}+X^{d+1}=e^{v}=1 / u\right\},{ }^{3}$ each slice $\Pi_{v}$ (or "horosphere") being an hyperbolic paraboloid:

$$
\left\{\begin{array}{c}
X^{\mu}=e^{v} x^{\mu}=\frac{1}{u} x^{\mu}, \quad \mu=0,1, \ldots, d-1, \\
X^{d}=\sinh v+\frac{1}{2} e^{v} x^{2}=\frac{1-u^{2}}{2 u}+\frac{1}{2 u} x^{2}, \\
x^{2}=x^{0^{2}}-x^{1^{2}}-\cdots-x^{d-1^{2}}, \\
X^{d+1}=\cosh v-\frac{1}{2} e^{v} x^{2}=\frac{1+u^{2}}{2 u}-\frac{1}{2 u} x^{2} .
\end{array}\right.
$$

\footnotetext{
${ }^{3}$ The coordinate $u=e^{-v}$ is frequently called $z$ in the recent literature. We are forced to change this notation because we reserve the letter $z$ to complex quantities. By allowing also negative values for $u$ the coordinate system (9) covers almost all the real manifold $A d S_{d+1}$.
} 
In each slice $\Pi_{v}, x^{0}, \ldots, x^{d-1}$ can be seen as coordinates of an event of a $d$-dimensional Minkowski spacetime $\mathbb{M}^{d}$ with metric $\mathrm{d} s_{M}^{2}=\mathrm{d} x^{0^{2}}-\mathrm{d} x^{1^{2}}-\cdots-\mathrm{d} x^{d-1^{2}}$ (here and in the following where it appears, an index $M$ stands for Minkowski). This explains why the horocyclic coordinates $(v, x)$ of the parametrization (9) are also called Poincaré coordinates. The scalar product (1) and the AdS metric can then be rewritten as follows:

$$
\begin{aligned}
& X \cdot X^{\prime}=\cosh \left(v-v^{\prime}\right)-\frac{1}{2} e^{v+v^{\prime}}\left(x-x^{\prime}\right)^{2}, \\
& \mathrm{~d} s_{\text {AdS }}^{2}=e^{2 v} \mathrm{~d} s_{M}^{2}-\mathrm{d} v^{2}=\frac{1}{u^{2}}\left(\mathrm{~d} s_{M}^{2}-\mathrm{d} u^{2}\right) .
\end{aligned}
$$

Eq. (10) implies that

$$
\left(X(v, x)-X\left(v, x^{\prime}\right)\right)^{2}=e^{2 v}\left(x-x^{\prime}\right)^{2} .
$$

This in turn implies that space-like separation in any slice $\Pi_{v}$ can be understood equivalently in the Minkowskian sense of the slice itself or in the sense of the ambient AdS universe.

Eq. (11) exhibits the region $\Pi$ of $A d S_{d+1}$ as a warped product [26] with warping function $\omega(v)=e^{v}$ and fibers conformal to $\mathbb{M}^{d}$. The use of this parametrization is crucial in a recent approach to the mass hierarchy problem [20] and to multidimensional cosmology. In this context the slices $\Pi_{v}$ are called branes. Finally, the representation of $\Pi$ by the parametrization (5) is specified by considering $\Pi$ as embedded in the fundamental sheet of $\widehat{A d S}_{d+1}$; it is therefore described by the following conditions on the coordinates $r, \tau$, e:

$$
-\pi<\tau<\pi ; \quad r \mathrm{e}^{d}+\sqrt{r^{2}+1} \cos \tau>0 .
$$

The "Euclidean" submanifold $E_{d+1}$ of $\widehat{A d S}_{d+1}^{(c)}$ is the set of all points $Z=X+i Y$ in $\widehat{A d S}_{d+1}^{(c)}$ such that $X=\left(0, X^{1}, \ldots, X^{d+1}\right), Y=\left(Y^{0}, 0, \ldots, 0\right)$ and $X^{d+1}>0$. It is therefore represented by the upper sheet (characterized by the condition $X^{d+1}>0$ ) of the two-sheeted hyperboloid with equation $X^{d+1^{2}}-Y^{0^{2}}-X^{1^{2}}-\cdots-X^{d^{2}}=1 . E_{d+1}$ is equally well represented in both parametrizations (5) and (9) as follows:

$$
Z=Z[r, \tau=i \sigma, \mathrm{e}] ; \quad(r, \sigma, \mathrm{e}) \in \mathbb{R} \times \mathbb{R} \times \mathbb{S}_{d-1}
$$

or

$$
Z=Z\left(v,\left(i y^{0}, x^{1}, \ldots, x^{d-1}\right)\right) ; \quad v \in \mathbb{R},\left(y^{0}, x^{1}, \ldots, x^{d-1}\right) \in \mathbb{R}^{d} .
$$

In view of (14), $E_{d+1}$ is contained in the fundamental sheet of $\widehat{A d S}(c)$.

For each $v$, the complexification $\Pi_{v}^{(c)}$ of the horosphere $\Pi_{v}$ is parametrized by formulae (6) in which $x$ is replaced by the complex Minkowskian vector $z=x+i y=\left(z^{0}, \ldots, z^{d}\right)$; the Euclidean submanifold of this complex Minkowskian manifold is obtained as the intersection $\Pi_{v}^{(c)} \cap E_{d+1}$.

\subsection{Quantum field theory}

Let us consider now a general QFT on $\widehat{A d S}_{d+1}$; for simplicity we limit the present discussion to one scalar field $\Phi(X)$. According to the general reconstruction procedure [15], 
a theory is completely determined by the set of all $n$-point vacuum expectation values (or "Wightman functions") of the field $\Phi$, given as distributions on the corresponding product manifolds $\left(\widehat{A d S}_{d+1}\right)^{n}$ :

$$
\mathcal{W}_{n}\left(X_{1}, \ldots X_{n}\right)=\left\langle\Omega, \Phi\left(X_{1}\right) \ldots, \Phi\left(X_{n}\right) \Omega\right\rangle .
$$

These distributions are supposed to be tempered when represented in the variables of the covering parametrization $X_{j}=X_{j}\left[r_{j}, \tau_{j}, \mathrm{e}_{j}\right]$ and to satisfy a set of general requirements which we will specify below.

Before doing it, we remark that a QFT on $\widehat{A d S}_{d+1}$ can be projected to a theory on $A d S_{d+1}$ itself if it is $2 \pi$-periodic in the time-parameter $\tau$, namely if each $\mathcal{W}_{n}\left(X_{1}, \ldots, X_{n}\right)$ is invariant under all individual substitutions $X_{j}\left[r_{j}, \tau_{j}, \mathrm{e}_{j}\right] \rightarrow X_{j}\left[r_{j}, \tau_{j}+2 \pi, \mathrm{e}_{j}\right]$.

An important class of fields, which can be explicitly constructed in a Fock space, is the class of "generalized free fields"; these fields are completely determined by their twopoint function $\mathcal{W}_{2}\left(X_{1}, X_{2}\right)$. In particular, the Klein-Gordon fields are those for which $\mathcal{W}_{2}\left(X_{1}, X_{2}\right)$ satisfies the corresponding field equation w.r.t. both points. Of course there are in general infinitely many inequivalent solutions to this problem (encoded in the choice of $\mathcal{W}_{2}$ ) and one has to select the meaningful ones on the basis of some physical principle; the existence of many possible theories even for a free field of a given mass is no surprise.

We shall assume that the distributions $\mathcal{W}_{n}$ satisfy the following properties: AdS invariance, positive-definiteness, hermiticity, local commutativity or microcausality, analyticity corresponding to an appropriate spectral condition and "dimensional boundary conditions" at infinity.

The requirement of AdS invariance (corresponding to the scalar character of the field) can be written as follows:

$$
\mathcal{W}_{n}\left(g X_{1}, \ldots, g X_{n}\right)=\mathcal{W}_{n}\left(X_{1}, \ldots, X_{n}\right), \quad \text { for any } g \in \widehat{G} .
$$

The usual positivity and hermiticity properties [15] are valid for scalar QFT's on any spacetime and we do not spell them out.

(a) Microcausality. $\Phi(X)$ commutes (as an operator-valued distribution) with $\Phi\left(X^{\prime}\right)$ for $X, X^{\prime}$ spacelike separated in the sense of the covering space $\widehat{A d S}_{d+1}$, as defined above (for theories in $A d S_{d+1}$ itself, it implies commutativity under the only condition $\left(X-X^{\prime}\right)^{2}<0$, which then includes a certain condition of commutativity at periodic time-like separations). As in the Minkowskian case, this postulate is equivalent to the coincidence of permuted Wightman functions at spacelike separation of consecutive arguments $X_{j}, X_{j+1}$ [15].

(b) Analyticity corresponding to energy spectrum condition. Since the parameter of the covering group of the rotations in the $(0, d+1)$-plane is interpreted as a genuine time-translation for the observers in all the corresponding Killing trajectories, and since the complexifications of these trajectories do not exhibit any geometrical periodicity 4 in $\widehat{A d S}_{d+1}^{(c)}$, it is legitimate to consider QFT's for which the corresponding infinitesimal generator $J_{0, d+1}$ is represented by a self-adjoint operator whose spectrum is bounded from

\footnotetext{
${ }^{4}$ Such geometrical periodicity in purely imaginary times gives rise to thermal effects for the corresponding observers, as it has already been checked in various examples of QFT on curved spacetimes [19,27-29].
} 
below: the latter postulate is in fact interpretable as a reasonable spectral condition on the energy, valid for all these observers. By using the standard Laplace transform argument $[15,36]$ in the corresponding time-variables $\tau_{1}, \ldots, \tau_{n}$, one is led to formulate this spectral condition by the following analyticity property of the Wightman functions:

Each tempered distribution $\mathcal{W}_{n}\left(X_{1}\left[r_{1}, \tau_{1}, \mathrm{e}_{1}\right], \ldots, X_{n}\left[r_{n}, \tau_{n}, \mathrm{e}_{n}\right]\right)$ is the boundary value of a holomorphic function $W_{n}\left(Z_{1}, \ldots, Z_{n}\right)$ which is defined in a complex neighborhood of the set

$$
\begin{aligned}
& \left\{Z=\left(Z_{1}, \ldots, Z_{n}\right) ; Z_{j}=X_{j}+i Y_{j} \in \widehat{A d S}_{d+1}^{(c)} ;\right. \\
& \left.\quad Z_{j}=Z_{j}\left[r_{j}, \tau_{j}, \mathrm{e}_{j}\right] ; \quad \operatorname{Im} \tau_{1}<\operatorname{Im} \tau_{2}<\cdots<\operatorname{Im} \tau_{n}\right\} .
\end{aligned}
$$

As a by-product, the Schwinger function $S_{n}$, that is the restriction of each $W_{n}$ to the Euclidean submanifold $\left\{\left(Z_{1}, \ldots, Z_{n}\right) \in\left(E_{d+1}\right)^{n} ; \sigma_{1}<\sigma_{2}<\cdots<\sigma_{n}\right\}$, is well-defined.

(c) Dimensional boundary conditions at infinity. In order to obtain relevant QFT's on the boundary of AdS spacetime (see Section 3), we are led to postulate a certain type of power-decrease at infinity for the Wightman functions which we call "dimensional boundary conditions at infinity"; such conditions can be shown to be valid in the case of Klein-Gordon fields (see Section 4).

By making use of the coordinates (5) we say that a QFT on $\widehat{A d S}_{d+1}$ is of asymptotic dimension $\Delta$ if the following limits exist in the sense of distributions:

$$
\begin{aligned}
& \lim _{\min \left(r_{1}, \ldots, r_{n}\right) \rightarrow+\infty}\left(r_{1} \cdots r_{n}\right)^{\Delta} \mathcal{W}_{n}\left(X_{1}\left[r_{1}, \tau_{1}, \mathrm{e}_{1}\right], \ldots, X_{n}\left[r_{n}, \tau_{n}, \mathrm{e}_{n}\right]\right) \\
& =\mathcal{W}_{n}^{\infty}\left(\left[\tau_{1}, \mathrm{e}_{1}\right], \ldots,\left[\tau_{n}, \mathrm{e}_{n}\right]\right) .
\end{aligned}
$$

We have to show that the above condition is meaningful, since it is not true in general that a distribution $\mathcal{W}_{n}\left(X_{1}, \ldots, X_{n}\right)$ can be restricted to the submanifold $\prod_{j=1}^{n} \widehat{C}_{r_{j}}$ of $\left(\widehat{A d S}_{d+1}\right)^{n}\left(C_{r}\right.$ was defined in Eq. (6)). Our spectral condition (b) implies that this can be done in the present framework. In fact, for each fixed $r_{1}, \ldots, r_{n}$ and $\mathrm{e}_{1}, \ldots, \mathrm{e}_{n}$, the existence of an analytic continuation $W_{n}$ of $\mathcal{W}_{n}$ in the variables $\tau_{1}, \ldots, \tau_{n}$ of the covering parametrization (5) in the tube domain $T_{n}=\left\{\left(\tau_{1}, \ldots, \tau_{n}\right) ; \operatorname{Im} \tau_{1}<\operatorname{Im} \tau_{2}<\cdots<\operatorname{Im} \tau_{n}\right\}$ implies that the boundary value of $W_{n}$ on the reals from this tube is a distribution in the variables $\tau_{1}, \ldots, \tau_{n}$ on each leaf obtained by fixing all the parameters $r_{j}$ and $\mathrm{e}_{j}$ and that it is even a regular (namely $C^{\infty}$ ) function of all these leaf parameters. The limit in Eq. (18) is therefore also defined as a distribution in the variables $\tau_{1}, \ldots, \tau_{n}$ with $C^{\infty}$ dependence with respect to the variables $\mathrm{e}_{j}$. Moreover, it is then natural to assume that the limit in Eq. (18) can be extrapolated to the holomorphic functions $W_{n}$ in their tube domains $T_{n}$ so that the corresponding limits $W_{n}^{\infty}$ are themselves holomorphic in $T_{n}$ and admit the corresponding distributions $\mathcal{W}_{n}^{\infty}$ as their boundary values on the reals. By restricting all these holomorphic functions to the Euclidean manifolds $\tau_{j}=i \sigma_{j}, j=1, \ldots, n$, one then obtains a similar condition for the Schwinger functions $S_{n}$ and the corresponding limits $S_{n}^{\infty}$.

If one wishes to select QFT's satisfying the property of uniqueness of the vacuum, one should supplement the previous requirements by an appropriate cluster property on the 
$n$-point functions. In order to obtain a relevant cluster property for the corresponding Lüscher-Mack CFT on the cone $\widehat{\mathcal{C}}_{2, d}$ obtained by the procedure described in our next section (namely the "conformal cluster property" described in [14]), one should formulate here a similar cluster property on the Schwinger functions on $\widehat{\operatorname{AdS}}{ }_{d+1}^{(c)}$, namely:

$$
\begin{aligned}
\lim _{\sigma \rightarrow+\infty} & \mathcal{W}_{m+n}\left(X_{1}\left[r_{1}, i \sigma_{1}, \mathrm{e}_{1}\right], \ldots, X_{m}\left[r_{m}, i \sigma_{m}, \mathrm{e}_{m}\right],\right. \\
X_{m+1} & {\left.\left[r_{m+1}, i\left(\sigma_{m+1}+\sigma\right), \mathrm{e}_{m+1}\right], \ldots, X_{m+n}\left[r_{m+n}, i\left(\sigma_{m+n}+\sigma\right), \mathrm{e}_{m+n}\right]\right) } \\
= & \mathcal{W}_{m}\left(X_{1}\left[r_{1}, i \sigma_{1}, \mathrm{e}_{1}\right], \ldots, X_{m}\left[r_{m}, i \sigma_{m}, \mathrm{e}_{m}\right]\right) \\
& \times \mathcal{W}_{n}\left(X_{m+1}\left[r_{m+1}, i \sigma_{m+1}, \mathrm{e}_{m+1}\right], \ldots, X_{m+n}\left[r_{m+n}, i \sigma_{m+n}, \mathrm{e}_{m+n}\right]\right) .
\end{aligned}
$$

\section{Causal quantum field theories on the manifolds $\widehat{C}_{r}$}

As a special application of the previous framework, it is meaningful to consider the restrictions of the distributions $\mathcal{W}_{n}$ to the submanifolds $\left(\widehat{C}_{r}\right)^{n}$ of $\left(\widehat{A d S}_{d+1}\right)^{n}$ (i.e., to the case when all variables $r_{j}$ are equal to $r$ ). One then notices that the positivity conditions satisfied by assumption by the distributions $\mathcal{W}_{n}$ on $\widehat{A d S}_{d+1}$ can be extended to test-functions of the variables $\tau_{j}$ and $\mathrm{e}_{j}$ localized in these submanifolds $r_{1}=\cdots=r_{n}=r$. In view of the standard reconstruction procedure [15], this allows one to say that in each slice $\widehat{C}_{r}$ the given field on $\widehat{A d S}_{d+1}$ yields by restriction a well-defined quantum field $\Phi_{r}(\tau, \mathrm{e})$. This field is obviously invariant under the product of the translation group with time-parameter $\tau$ by the orthogonal group $S O(d)$ of space transformations acting on the sphere $\mathbb{S}_{d-1}$ of the variables e. Moreover, it follows from the microcausality postulate (a) together with Eqs. (7) and (8) that the field $\Phi_{r}$ also satisfies local commutativity in the sense of the spacetime manifold $\widehat{C}_{r}$. Finally, in view of (b), the $n$-point functions of $\Phi_{r}$ are (for each $r$ ) boundary values of holomorphic functions of the complex variables $\tau_{1}, \ldots, \tau_{n}$ in the tube $T_{n}$, which shows that these theories satisfy a spectral condition with respect to the generator of timetranslations.

\section{Correspondence with conformal field theories on $\widehat{\mathcal{C}_{2, d}}$ à la Lüscher-Mack}

We shall now introduce the asymptotic cone $\mathcal{C}_{2, d}$ (respectively, $\mathcal{C}_{2, d}^{(c)}$ ) of $A d S_{d+1}$ (respectively, $A d S_{d+1}^{(c)}$ ) and wish to identify the limit (in the sense of Eq. (18)) of a QFT on $\widehat{A d S}_{d+1}$ satisfying the previous properties with a QFT on the corresponding covering $\widehat{\mathcal{C}_{2, d}}$ of $\mathcal{C}_{2, d}$. To do this, we first notice that by adapting the covering parametrization (5) of $\widehat{A d S}_{d+1}$ to the case of its asymptotic cone, $\mathcal{C}_{2, d}=\left\{\eta=\left(\eta^{0}, \ldots, \eta^{(d+1)}\right) ; \eta^{0^{2}}-\eta^{1^{2}}-\right.$ $\left.\cdots-\eta^{d^{2}}+\eta^{d+1^{2}}=0\right\}$, one readily obtains the following parametrization (with the same notations as [14], but in dimension $d+2$ ):

$$
\left\{\begin{array}{l}
\eta^{0}=r \sin \tau \\
\eta^{i}=r \mathrm{e}^{i}, \quad i=1, \ldots, d \\
\eta^{d+1}=r \cos \tau
\end{array}\right.
$$


with $\mathrm{e}^{1^{2}}+\cdots+\mathrm{e}^{d^{2}}=1$ and $r \geqslant 0$, or in brief: $\eta=\eta[r, \tau, \mathrm{e}]$.

The parametrization (20) allows one to introduce the coverings $\widehat{\mathcal{C}}_{2, d}$ and $\widehat{\mathcal{C}}_{2, d}^{(c)}$ of $\mathcal{C}_{2, d}$ and $\mathcal{C}_{2, d}^{(c)}$ by again unfolding the $2 \pi$-periodic coordinate $\tau$ (respectively, $\operatorname{Re} \tau$ ). A privileged "fundamental sheet" is defined on these coverings by imposing the condition $-\pi<\tau<\pi$ (respectively, $-\pi<\operatorname{Re} \tau<\pi$ ).

We also note that the standard condition of spacelike separation on $\mathcal{C}_{2, d}$ is similar to the condition chosen on the AdS spacetime, namely

$$
\left(\eta-\eta^{\prime}\right)^{2}=r^{2}\left[4\left(\sin \left(\frac{\tau-\tau^{\prime}}{2}\right)\right)^{2}-\left(\mathrm{e}-\mathrm{e}^{\prime}\right)^{2}\right]=-2 r^{2}\left(\cos \left(\tau-\tau^{\prime}\right)-\mathrm{e} \cdot \mathrm{e}^{\prime}\right)<0,
$$

and yields the corresponding global causal ordering on $\widehat{\mathcal{C}}_{2, d}$

$$
(\tau, \mathrm{e})>\left(\tau^{\prime}, \mathrm{e}^{\prime}\right) \quad \text { iff } \tau-\tau^{\prime}>2 \operatorname{Arcsin}\left(\frac{\left(\mathrm{e}-\mathrm{e}^{\prime}\right)^{2}}{4}\right)^{1 / 2},
$$

equivalently written, e.g., in [14] as $\tau-\tau^{\prime}>\operatorname{Arccos}\left(\mathrm{e} \cdot \mathrm{e}^{\prime}\right)$. Note that in the space of variables $\left(\tau, \tau^{\prime}, \mathrm{e}, \mathrm{e}^{\prime}\right)$, the region described by Eq. (22) is exactly the limit of the region given by Eq. (8) when $r$ tends to infinity.

By taking the intersection of $\mathcal{C}_{2, d}$ with the family of hyperplanes with equation $\eta^{d}+$ $\eta^{d+1}=e^{v}$, one obtains the analogue of the horocyclic parametrization (9), namely:

$$
\left\{\begin{array}{l}
\eta^{\mu}=e^{v} x^{\mu}, \quad \mu=0,1, \ldots, d-1, \\
\eta^{d}=\frac{1}{2} e^{v}\left(1+x^{2}\right), \quad x^{2}=x^{0^{2}}-x^{1^{2}}-\cdots-x^{d-1^{2}}, \\
\eta^{d+1}=\frac{1}{2} e^{v}\left(1-x^{2}\right),
\end{array}\right.
$$

which implies the following identity (similar to (10)) between quadratic forms:

$$
\left(\eta-\eta^{\prime}\right)^{2}=e^{v+v^{\prime}}\left(x-x^{\prime}\right)^{2} .
$$

By taking Eqs. (20) into account, one then sees that these formulae correspond (in dimension $d$ ) to the embedding of Minkowski space into the covering of the cone $\mathcal{C}_{2, d}$ (see [30] and references therein), namely one has (in view of the identification $\eta^{d}+\eta^{d+1}=$ $\left.e^{v}=r\left(\mathrm{e}^{d}+\cos \tau\right)\right)$ :

$$
x^{0}=\frac{\sin \tau}{\cos \tau+\mathrm{e}^{d}}, \quad x^{i}=\frac{\mathrm{e}^{i}}{\cos \tau+\mathrm{e}^{d}},
$$

with

$$
\cos \tau+\mathrm{e}^{d}>0, \quad-\pi<\tau<\pi .
$$

Let us now consider a general QFT on $\widehat{A d S}_{d+1}$ whose Wightman functions $\mathcal{W}_{n}$ satisfy AdS invariance together with the properties (a)-(c) described in the previous section. In view of (c), we can associate with the latter the following set of $n$-point distributions $\widetilde{\mathcal{W}}_{n}\left(\eta_{1}, \ldots, \eta_{n}\right)$ on $\widehat{\mathcal{C}_{2, d}}$ :

$$
\tilde{\mathcal{W}}_{n}\left(\eta_{1}, \ldots, \eta_{n}\right)=\left(r_{1} \cdots r_{n}\right)^{-\Delta} \mathcal{W}_{n}^{\infty}\left(\left[\tau_{1}, \mathrm{e}_{1}\right], \ldots,\left[\tau_{n}, \mathrm{e}_{n}\right]\right) .
$$


At first, one can check that the set of distributions $\widetilde{\mathcal{W}}_{n}$ satisfy the required positivity conditions for defining a QFT on $\widehat{\mathcal{C}_{2, d}}$. This is because, in view of postulate (c) (applied with all $r_{j}$ equal to the same $r$ ), the distributions $\mathcal{W}_{n}^{\infty}$ appear as the limits of the $n$-point functions of the QFT's on the spacetimes $\widehat{C}_{r}$ when $r$ tends to infinity. The positivity conditions satisfied by the latter are then preserved in the limit, in terms of test-functions of the variables $\tau_{j}$ and $\mathrm{e}_{j}$, and then extended in a trivial way into the radial variables $r_{j}$ as positivity conditions for the distributions on the cone $\widehat{\mathcal{C}_{2, d}}$ (by using the appropriate test-functions homogeneous in the variables $r_{j}[14]$ ).

It follows from the reconstruction procedure [15] that the set of distributions $\widetilde{\mathcal{W}}_{n}$ define a quantum field $\widetilde{O}(\eta)$ on $\widehat{\mathcal{C}_{2, d}} . \widetilde{O}(\eta)$ enjoys the following properties:

Microcausality. Since the region (22) is the limit of (8) for $r$ tending to infinity, it results from the boundary condition (c) and from the local commutativity of all fields $\Phi_{r}$ in the corresponding spacetimes $\widehat{C}_{r}$ that the field $\widetilde{O}(\eta)$ satisfies local commutativity on $\widehat{C_{2, d}}$.

Spectral condition. In view of our postulate (c) extended to the complex domain $T_{n}$ in the variables $\tau$, we see that the $n$-point distributions $\tilde{\mathcal{W}}_{n}\left(\eta_{1}, \ldots, \eta_{n}\right)$ are boundary values of holomorphic functions in the same analyticity domains of $\left(\widehat{\mathcal{C}}_{2, d}^{(c)}\right)^{n}$ as those of the Lüscher-Mack field theories [14]. In particular, the restrictions of these holomorphic functions to the Euclidean space domains $\left\{\eta=\left(\eta_{1}, \ldots, \eta_{n}\right) ; \eta_{j}^{0}=i r \sinh \sigma_{j}, \eta_{j}^{i}=r \mathrm{e}_{j}^{i}, i=\right.$ $\left.1, \ldots, d, \eta_{j}^{d+1}=r \cosh \sigma_{j} ; \sigma_{1}<\sigma_{2}<\cdots<\sigma_{n}\right\}$ yield the Schwinger functions of the theory. It is also clear that, if the original Schwinger functions on the complexified AdS space satisfy the cluster property (19), the corresponding Schwinger functions on $\widehat{\mathcal{C}}_{2, d}^{(c)}$ satisfy the Luscher-Mack conformal cluster property (formula (5.1) of [14]) ensuring the uniqueness of the vacuum.

We are now going to establish that the $\widehat{G}$-invariance (17) of the AdS $n$-point functions, together with the properties (a)-(c), imply the conformal invariance of the field $\widetilde{O}(\eta)$; more precisely, we wish to show that the Wightman functions $\tilde{\mathcal{W}}_{n}$ of this field are invariant under the action on $\widehat{\mathcal{C}_{2, d}}$ of the group $\widehat{G}$, now interpreted as in [14] as the "quantum mechanical conformal group", namely that one has:

$$
\tilde{\mathcal{W}}_{n}\left(g \eta_{1}, \ldots, g \eta_{n}\right)=\tilde{\mathcal{W}}_{n}\left(\eta_{1}, \ldots, \eta_{n}\right)
$$

for all $g$ in $\widehat{G}$.

A part of this invariance is trivial in view of the limiting procedure of (c): it is the invariance under the rotations in the $(0, d+1)$-plane (i.e., the translations in the time variables $\tau$ ) and the invariance under the spatial orthogonal group of the subspace of variables $\left(\eta^{1}, \ldots, \eta^{d}\right)$ (acting on the sphere $\left.\mathbb{S}_{d-1}\right)$.

In order to show that the invariance condition (28) holds for all $g$ in $\widehat{G}$, it remains to show that it holds for all one-parameter subgroups of pseudo-rotations in the $(0, i)$-planes and in the $(i, d+1)$-planes of coordinates, with $i=1, \ldots, d$. Let us consider the first case with, e.g., $i=1$ and associate with the corresponding subgroup $G_{0,1}$ of pseudo-rotations the following parametrizations $X=X\{\rho, \psi, u\}$ and $\eta=\eta\{\rho, \psi, u\}$ (with $u=\left(u^{2}, \ldots, u^{d+1}\right)$ ) of $\widehat{A d S}_{d+1}$ and of $\widehat{\mathcal{C}}_{2, d}$ : 


$$
\begin{aligned}
& \left\{\begin{array}{l}
X^{0}=\rho \sinh \psi, \\
X^{1}=\rho \cosh \psi \\
X^{i}=\sqrt{\rho^{2}+1} u^{i}, \quad i=2, \ldots, d, \\
X^{d+1}=\sqrt{\rho^{2}+1} u^{d+1}, \quad u^{d+1^{2}}-u^{2^{2}}-\cdots-u^{d^{2}}=1,
\end{array}\right. \\
& \left\{\begin{array}{l}
\eta^{0}=\rho \sinh \psi, \\
\eta^{1}=\rho \cosh \psi, \\
\eta^{i}=\rho, u^{i}, \quad i=2, \ldots, d, \\
\eta^{d+1}=\rho u^{d+1}, \quad u^{d+1^{2}}-u^{2^{2}}-\cdots-u^{d^{2}}=1 .
\end{array}\right.
\end{aligned}
$$

For $g \in G_{0,1}$, the invariance condition (28) to be proven can be written as follows (with the simplified notation $\left.\widetilde{\mathcal{W}}_{n}\left(\eta_{1}, \ldots, \eta_{n}\right)=\widetilde{\mathcal{W}}_{n}\left(\eta_{j}\right)\right)$ :

$$
\tilde{\mathcal{W}}_{n}\left(\eta_{j}\left\{\rho_{j}, \psi_{j}+a, u_{j}\right\}\right)=\tilde{\mathcal{W}}_{n}\left(\eta_{j}\left\{\rho_{j}, \psi_{j}, u_{j}\right\}\right)
$$

for all real $a$. Now in view of the definition (27) of $\tilde{\mathcal{W}}_{n}\left(\eta_{j}\right)$ and of the relations between the sets of parameters $(r, \tau, \mathrm{e})$ and $(\rho, \psi, u)$ obtained by identification of the expressions (20) and (30) of $\eta$, the invariance condition (31) to be proven is equivalent to the following condition for the asymptotic forms of the AdS $n$-point functions $\mathcal{W}_{n}^{\infty}$ (for all $a$ ):

$$
\begin{gathered}
\prod_{1 \leqslant k \leqslant n}\left(\left(\sinh \psi_{k}\right)^{2}+\left(u_{k}^{d+1}\right)^{2}\right)^{-\Delta / 2}\left(\left(\sinh \left(\psi_{k}+a\right)\right)^{2}+\left(u_{k}^{d+1}\right)^{2}\right)^{\Delta / 2} \\
\times \mathcal{W}_{n}^{\infty}\left(\left[\operatorname{arctg} \frac{\sinh \psi_{j}}{u_{j}^{d+1}}, \frac{\cosh \psi_{j}}{\left(\left(\sinh \psi_{j}\right)^{2}+\left(u_{j}^{d+1}\right)^{2}\right)^{1 / 2}}, \frac{u_{j}^{i}}{\left(\left(\sinh \psi_{j}\right)^{2}+\left(u_{j}^{d+1}\right)^{2}\right)^{1 / 2}}\right]\right) \\
=\mathcal{W}_{n}^{\infty}\left(\left[\operatorname{arctg} \frac{\sinh \left(\psi_{j}+a\right)}{u_{j}^{d+1}}, \frac{\cosh \psi_{j}}{\left(\left(\sinh \left(\psi_{j}+a\right)\right)^{2}+\left(u_{j}^{d+1}\right)^{2}\right)^{1 / 2}},\right.\right. \\
\left.\left.\times \frac{u_{j}^{i}}{\left(\left(\sinh \left(\psi_{j}+a\right)\right)^{2}+\left(u_{j}^{d+1}\right)^{2}\right)^{1 / 2}}\right]\right) .
\end{gathered}
$$

In this equation the symbol $\operatorname{arctg}(\cdot)$ denotes the angle $\tau_{j}$ of the parametrization (20), which can take all real values; however, one notices that under the transformation $\psi_{j} \rightarrow \psi_{j}+a$, the angle $\tau_{j}$ varies in such a way that the point $\eta$ remains in the same sheet of the covering $\widehat{\mathcal{C}_{2, d}}$ of the cone $\mathcal{C}_{2, d}$ (e.g., $-\pi / 2<\tau_{j}<\pi / 2$ for the choice of Arctg).

Comparing the parametrizations (5) and (29) of $\widehat{A d S}_{d+1}$ we obtain the following relations:

$$
\begin{aligned}
r & =\rho\left[(\sinh \psi)^{2}+\left(u^{d+1}\right)^{2}+\frac{\left(u^{d+1}\right)^{2}-1}{\rho^{2}}\right]^{1 / 2} \equiv \rho h_{\rho}\left(\psi, u^{d+1}\right), \\
\tau & =\operatorname{arctg}\left[\left(1+\frac{1}{\rho^{2}}\right)^{-1 / 2} \frac{\sinh \psi}{u^{d+1}}\right], \\
\mathrm{e}^{1} & =\frac{\cosh \psi}{h_{\rho}\left(\psi, u^{d+1}\right)}, \quad \mathrm{e}^{i}=\frac{u^{i}}{h_{\rho}\left(\psi, u^{d+1}\right)}\left(1+\frac{1}{\rho^{2}}\right)^{1 / 2} .
\end{aligned}
$$

Note that the function $h_{\rho}$ introduced in (33) is such that

$$
\lim _{\rho \rightarrow \infty} h_{\rho}\left(\psi, u^{d+1}\right)=\left[(\sinh \psi)^{2}+\left(u^{d+1}\right)^{2}\right]^{1 / 2} .
$$


This implies that it is equivalent to take the limits in Eq. (18) for $\rho_{j}$ (instead of $r_{j}$ ) tending to infinity and at fixed value of $\psi_{j}$ and $u_{j}$, after plugging the expressions (33)-(35) of $r_{j}, \tau_{j}, \mathrm{e}_{j}$ into both sides of Eq. (18):

$$
\begin{aligned}
& \lim _{\min \left(\rho_{1}, \ldots, \rho_{n}\right) \rightarrow+\infty} \mid\left(\rho_{1} \cdots \rho_{n}\right)^{\Delta} \mathcal{W}_{n}\left(X_{j}\left\{\rho_{j}, \psi_{j}, u_{j}\right\}\right)-\prod_{1 \leqslant k \leqslant n} h_{\rho_{k}}\left(\psi_{k}, u_{k}^{d+1}\right)^{-\Delta} \\
& \quad \times \mathcal{W}_{n}^{\infty}\left(\left[\operatorname{arctg}\left[\frac{\sinh \psi_{j}}{\left(1+\frac{1}{\rho_{j}^{2}}\right)^{1 / 2} u_{j}^{d+1}}\right], \frac{\cosh \psi_{j}}{h_{\rho_{j}}\left(\psi_{j}, u_{j}^{d+1}\right)}, \frac{u_{j}^{i}\left(1+\frac{1}{\rho_{j}^{2}}\right)^{1 / 2}}{h_{\rho_{j}}\left(\psi_{j}, u_{j}^{d+1}\right)}\right]\right) \mid=0 .
\end{aligned}
$$

If we now also consider the vanishing limit of the same difference after the transformation $\psi_{j} \rightarrow \psi_{j}+a$ has been applied, and take into account the fact that, by assumption, the first term of this difference has remained unchanged, we obtain the following relation:

$$
\begin{aligned}
& \lim _{\min \left(\rho_{1}, \ldots, \rho_{n}\right) \rightarrow+\infty} \mid \prod_{1 \leqslant k \leqslant n} h_{\rho_{k}}\left(\psi_{k}, u_{k}^{d+1}\right)^{-\Delta} \\
& \quad \times \mathcal{W}_{n}^{\infty}\left(\left[\operatorname{arctg}\left[\frac{\sinh \psi_{j}}{\left(1+\frac{1}{\rho_{j}^{2}}\right)^{1 / 2} u_{j}^{d+1}}\right], \frac{\cosh \psi_{j}}{h_{\rho_{j}}\left(\psi_{j}, u_{j}^{d+1}\right)}, \frac{u_{j}^{i}\left(1+\frac{1}{\rho_{j}^{2}}\right)^{1 / 2}}{h_{\rho_{j}}\left(\psi_{j}, u_{j}^{d+1}\right)}\right]\right) \\
& -\prod_{1 \leqslant k \leqslant n} h_{\rho_{j}}\left(\psi_{k}+a, u_{k}^{d+1}\right)^{-\Delta} \\
& \quad \times \mathcal{W}_{n}^{\infty}\left(\left[\operatorname{arctg}\left[\frac{\sinh \left(\psi_{j}+a\right)}{\left(1+\frac{1}{\rho_{j}^{2}}\right)^{1 / 2} u_{j}^{d+1}}\right], \frac{\left(\cosh \psi_{j}+a\right)}{h_{\rho_{j}}\left(\psi_{j}+a, u_{j}^{d+1}\right)}, \frac{u_{j}^{i}\left(1+\frac{1}{\rho_{j}^{2}}\right)^{1 / 2}}{h_{\rho_{j}}\left(\psi_{j}+a, u_{j}^{d+1}\right)}\right]\right) \mid=0 .
\end{aligned}
$$

Now it is easily seen that in the latter, the limit can be taken separately in each term and that the resulting equality yields precisely the required covariance relation (32) for $\mathcal{W}_{n}^{\infty}$.

Although the previous formulae have been written in terms of the distributions $\mathcal{W}_{n}$ and of their asymptotic forms, one could reproduce the argument in a completely rigorous way [17] in terms of the functions $W_{n}$ in the tube domains $T_{n}$ of the variables $\tau_{j}$, all the functions involved being then of class $\mathcal{C}^{\infty}$ with respect to all the variables $\left(\rho_{j}, \psi_{j}, u_{j}\right)$ and all the limits being taken in the sense of regular functions; the covariance relations on the reals will then be obtained as relations for the corresponding boundary values (in the sense of distributions). The treatment of the covariance with respect to the pseudo-rotation groups $G_{i, d+1}$ is completely similar.

We can then summarize the results of this section by the following statement:

the procedure we have described (expressed by Eqs. (18) and (27)) displays a general AdS CFT correspondence for QFT's:

$$
\Phi(X) \rightarrow \widetilde{\mathcal{O}}(\eta)
$$


between a scalar (AdS invariant) quantum field $\Phi(X)$ on the covering $\widehat{A d S}_{d+1}$ of $A d S_{d+1}$ whose Wightman functions satisfy the properties $(a)-(c)$, and a conformally invariant local (i.e., causal) field $\widetilde{\mathcal{O}}(\eta)$ on the covering $\widehat{\mathcal{C}_{2, d}}$ of the cone $\mathcal{C}_{2, d}$, enjoying the LüscherMack spectral condition; the degree of homogeneity (dimension) $\Delta$ of $\tilde{\mathcal{O}}(\eta)$ is equal to the asymptotic dimension of the AdS field $\Phi(X)$.

Of course, from this general point of view, the correspondence may a priori be manyto-one. Finally, according to the formalism described in $[14,30]$, the correspondence (39) can be completed by saying that there exists a unique conformal (Minkowskian) local field $\mathcal{O}(x)$ of dimension $\Delta$ whose $n$-point functions $\mathcal{W}_{n}^{M}$ are expressed in terms of those of $\widetilde{\mathcal{O}}(\eta)$ by the following formulae:

$$
\begin{aligned}
\mathcal{W}_{n}^{M}\left(x_{1}, \ldots, x_{n}\right) & =e^{\left(v_{1}+\cdots+v_{n}\right) \Delta} \tilde{\mathcal{W}}_{n}\left(\eta_{1}, \ldots, \eta_{n}\right) \\
& =\Pi_{1 \leqslant j \leqslant n}\left(\eta_{j}^{d}+\eta_{j}^{d+1}\right)^{\Delta} \tilde{\mathcal{W}}_{n}\left(\eta_{1}, \ldots, \eta_{n}\right) .
\end{aligned}
$$

In the latter, the Minkowskian variables $x_{j}$ are expressed in terms of the cone variables $\eta_{j}$ by inverting (23), which yields:

$$
x_{j}^{\mu}=\frac{\eta_{j}^{\mu}}{\eta_{j}^{d}+\eta_{j}^{d+1}} \text {. }
$$

\section{Two-point functions}

\subsection{The analytic structure of two-point functions on the AdS spacetime}

It turns out that in all field theories on $\widehat{A d S}_{d+1}$ satisfying the general requirements described in Section 2.2, the two-point function enjoys maximal analyticity properties in all the coordinates, as it is the case for the Minkowski [15] and de Sitter cases [19]. A full proof of these results will be found in [17]. We shall only give here a descriptive account of them, needed for further applications. Since, in particular, AdS covariance and the "energy spectrum condition" (b) of Section 2.2 are responsible for this maximal analytic structure and since (as seen below) the latter determines completely satisfactory solutions for the case of Klein-Gordon AdS fields, we shall consider this general class of two-point functions as "preferred".

There are two distinguished complex domains [17] of $A d S_{d+1}^{(c)}$, invariant under real AdS transformations, which are of crucial importance for a full understanding of the structures associated with two-point functions. They are given by:

$$
\begin{aligned}
& T^{+}=\left\{Z=X+i Y \in A d S_{d+1}^{(c)} ; Y^{2}>0, \epsilon(Z)=+1\right\}, \\
& T^{-}=\left\{Z=X+i Y \in A d S_{d+1}^{(c)} ; Y^{2}>0, \epsilon(Z)=-1\right\},
\end{aligned}
$$

where

$$
\epsilon(Z)=\operatorname{sign}\left(Y^{0} X^{d+1}-X^{0} Y^{d+1}\right) .
$$


$T^{+}$and $T^{-}$are the AdS version of the usual forward and backward tubes $T_{M}^{+}$and $T_{M}^{-}$of complex Minkowski spacetime, obtained in correspondence with the energy-momentum spectrum condition [15]; let us recall their definition (in arbitrary spacetime dimension $p$ ):

$$
\begin{aligned}
& T_{M}^{+}=\left\{z=x+i y \in \mathbb{M}^{p(c)} ; y^{2}>0, y^{0}>0\right\}, \\
& T_{M}^{-}=\left\{z=x+i y \in \mathbb{M}^{p(c)} ; y^{2}>0, y^{0}<0\right\} .
\end{aligned}
$$

In the same way as these Minkowskian tubes are generated by the action of real Lorentz transformations on the "flat" (one complex time-variable) domains $\{z=x+i y ; \quad y=$ $\left(y^{0}, \overrightarrow{0}\right) ; y^{0}>0$ (respectively, $\left.\left.y^{0}<0\right)\right\}$, the domains (42) of $A d S_{d+1}^{(c)}$ are generated by the action of the group $G$ on the flat domains obtained by letting $\tau$ vary in the half-planes $\operatorname{Im} \tau>0$ or $\operatorname{Im} \tau<0$ and keeping $r$ and $e$ real in the covering parametrization (5) of the AdS quadric. In fact, by using the complex extension of this parametrization and putting $r=\sinh (\psi+i \phi), \tau=\operatorname{Re} \tau+i \sigma$ one can represent the domains (42) by the following semi-tubes (invariant under translations in the variable $\operatorname{Re} \tau$ ):

$$
\pm \sinh \sigma>\left[\frac{(\sin \phi)^{2}+\left((\cosh \psi)^{2}-(\cos \phi)^{2}\right)(\operatorname{Im} e)^{2}}{(\cosh \psi)^{2}-(\sin \phi)^{2}}\right]^{1 / 2} .
$$

This representation (which clearly contains the previously mentioned flat domains) can be thought of, either as representing the domains (42) of $A d S^{(c)}$ if $\tau$ is identified to $\tau+2 \pi$, or coverings of the latter embedded in $\widehat{A d S} S_{d+1}^{(c)}$, which we denote by $\widehat{T}^{+}$and $\widehat{T}^{-}$, if one does not make this identification.

One typical property of Wightman's QFT [15] is that any two-point distribution $\mathcal{W}_{M}\left(x, x^{\prime}\right)$ satisfying the spectral condition is the boundary value of a function $W_{M}\left(z, z^{\prime}\right)$ holomorphic for $z \in T_{M}^{-}$and $z^{\prime} \in T_{M}^{+}$. An analogous property also holds for $n$-point functions.

It is a consequence of AdS invariance together with the spectrum assumption (b) [17] that, also in the AdS spacetime, general two-point functions can be characterized by the following global analyticity property which plays the role of a $G$-invariant spectral condition:

$\left(\mathrm{b}^{\text {(inv) })}\right.$ Normal analyticity condition for two-point functions: the two-point function $\mathcal{W}\left(X, X^{\prime}\right)$ is the boundary value of a function $W\left(Z, Z^{\prime}\right)$ which is holomorphic in the domain $\widehat{T}^{-} \times \widehat{T}^{+}$of $\widehat{A d S}_{d+1}^{(c)} \times \widehat{A d S}(c)$.

A further use of AdS invariance implies that $W\left(Z, Z^{\prime}\right)$ is actually a function $w(\zeta)$ of a single complex variable $\zeta$; this variable $\zeta$ can be identified with $Z \cdot Z^{\prime}$ when $Z$ and $Z^{\prime}$ are both in the fundamental sheet of $\widehat{A d S}_{d+1}^{(c)}$; AdS invariance and the normal analyticity condition together imply the following

Maximal analyticity property. $w(\zeta)$ is analytic in the covering $\widehat{\Theta}$ of the cut-plane $\Theta=\{\mathbb{C} \backslash[-1,1]\}$.

For special theories which are periodic in the time coordinate $\tau, w(\zeta)$ is in fact analytic in $\Theta$ itself. One can now introduce all the usual Green functions. The "permuted Wightman function" $\mathcal{W}\left(X^{\prime}, X\right)=\left\langle\Omega, \Phi\left(X^{\prime}\right) \Phi(X) \Omega\right\rangle$ is the boundary value of $W\left(Z, Z^{\prime}\right)$ from 
the domain $\left\{\left(Z, Z^{\prime}\right): Z \in \widehat{T}^{+}, Z^{\prime} \in \widehat{T}^{-}\right\}$. The commutator function is then $\mathcal{C}\left(X, X^{\prime}\right)=$ $\mathcal{W}\left(X, X^{\prime}\right)-\mathcal{W}\left(X^{\prime}, X\right)$. The retarded propagator $\mathcal{R}\left(X, X^{\prime}\right)$ is introduced by splitting the support of the commutator $\mathcal{C}\left(X, X^{\prime}\right)$ as follows

$$
\mathcal{R}\left(X, X^{\prime}\right)=i \theta\left(\tau-\tau^{\prime}\right) \mathcal{C}\left(X, X^{\prime}\right) .
$$

The other Green functions are then defined in terms of $\mathcal{R}$ by the usual formulae: the advanced propagator is given by $\mathcal{A}=\mathcal{R}-i \mathcal{C}$ while the chronological propagator is given by $\mathcal{F}=-i \mathcal{A}+\mathcal{W}$.

Note finally that, as a function of the single variable $\zeta=X \cdot X^{\prime}$, the jump $i \delta w(\zeta)$ of $i w(\zeta)$ across its cut $(-\infty,+1]$ coincides with the retarded propagator $R\left(X, X^{\prime}\right)$ (or the advanced one); in the periodic (i.e., "true AdS") case, the support of $\delta w$ reduces to the compact interval $[-1,+1]$.

\subsection{The simplest example revisited: Klein-Gordon fields in the AdS/CFT correspondence}

The Wightman functions of fields satisfying the Klein-Gordon equation $A d S_{d+1}$

$$
\square_{A d S} \Phi+m^{2} \Phi=0
$$

display the simplest example of the previous analytic structure:

$$
W_{\nu}\left(Z, Z^{\prime}\right)=w_{\nu}(\zeta)=\frac{e^{-i \pi \frac{d-1}{2}}}{(2 \pi)^{\frac{d+1}{2}}}\left(\zeta^{2}-1\right)^{-\frac{d-1}{4}} Q_{\nu-\frac{1}{2}}^{\frac{d-1}{2}}(\zeta)
$$

Here $Q$ is a second-kind Legendre's function ${ }^{5}$ [31]; the parameter $v$ is linked to the field's mass by the relation

$$
v^{2}=\frac{d^{2}}{4}+m^{2}
$$

and the normalization of $W_{v}$ is chosen by imposing the short-distance Hadamard behavior.

Since $W_{v}\left(Z, Z^{\prime}\right)$ and $W_{-v}\left(Z, Z^{\prime}\right)$ are solutions of the same Klein-Gordon equation (and share the same analyticity properties), the question arises if these Wightman function both define acceptable QFT's on $A d S_{d+1}$. The answer [21] is that only theories with $v \geqslant-1$ are acceptable and there are therefore two regimes: for $v>1$ there is only one field theory corresponding to a given mass while for $|\nu|<1$ there are two theories. The case $v=1$ is a limit case. Eq. (48) shows clearly that the only difference between the theories parametrized by opposite values of $v$ is in their large distance behavior. More precisely, in view of Eq. (3.3.1.4) of [31], we can write:

$$
w_{-v}(\zeta)=w_{\nu}(\zeta)+\frac{\sin \pi v}{(2 \pi)^{\frac{d+1}{2}}} \Gamma\left(\frac{d}{2}-v\right) \Gamma\left(\frac{d}{2}+v\right)\left(\zeta^{2}-1\right)^{-\frac{d-1}{4}} P_{-\frac{1}{2}-v}^{-\frac{d-1}{2}}(\zeta)
$$

\footnotetext{
${ }^{5}$ This is the way these Wightman functions were first written in [7] for the four-dimensional case $d=3$. Their identification with second-kind Legendre functions is worth being emphasized, in place of their less specific (although exact) introduction under the general label of hypergeometric functions, used in recent papers. In fact Legendre functions are basically linked to the geometry of the dS and AdS quadrics from both group-theoretical and complex analysis viewpoints [19,23-25].
} 
Now we notice that in this relation (where all terms are solutions of the same Klein-Gordon equation) the last term is regular on the cut $\zeta \in[-1,1]$. This entails (reintroducing the AdS radius $R$ ) that, in the two theories, the $c$-number commutator $\left[\Phi(X), \Phi\left(X^{\prime}\right)\right]$ takes the same value for all (time-like separated) vectors $\left(X, X^{\prime}\right)$ such that $\left|X \cdot X^{\prime}\right|<R^{2}$. Therefore we can say that the two theories represent the same algebra of local observables at short distances (with respect to the radius $R$ ). But since the last term in the latter relation grows the faster the larger is $|v|$ (see [31] Eqs. (3.9.2)), we see that the two theories drastically differ by their long range behaviors.

The existence of the two regimes above has given rise to two distinct treatments of the AdS/CFT correspondence in the two cases [32] and symmetry breaking had been advocated to explain the difference.

In the present context, by applying the correspondence as given in Eq. (39), the two regimes can be treated in one stroke. Indeed, Eq. (3.9.2.21) of [31] reports the following large $\zeta$ behavior of the Legendre's function $Q$ (valid for any complex $v$ ):

$$
Q_{\nu-\frac{1}{2}}^{\frac{d-1}{2}}(\zeta) \simeq e^{i \pi \frac{d-1}{2}} 2^{-v-\frac{1}{2}} \frac{\Gamma\left(v+\frac{d}{2}\right)}{\Gamma(v+1)} \pi^{\frac{1}{2}} \zeta^{-\frac{1}{2}-v} .
$$

It follows that the two-point function (48) and thereby all the $n$-point functions of the corresponding Klein-Gordon field satisfy the dimensional boundary conditions at infinity with dimension $\Delta=\frac{d}{2}+v$. Indeed, let $\tau$ and $\tau^{\prime}$ be complex and such that $\operatorname{Im} \tau<\operatorname{Im} \tau^{\prime}$. It follows that

$$
\begin{aligned}
W_{\nu}^{\infty}\left([\tau, \mathrm{e}],\left[\tau^{\prime}, \mathrm{e}^{\prime}\right]\right) & =\lim _{r, r^{\prime} \rightarrow \infty}\left(r r^{\prime}\right)^{\frac{d}{2}+v} W_{\nu}\left(Z[\tau, r, \mathrm{e}], Z^{\prime}\left[\tau^{\prime}, r^{\prime}, \mathrm{e}^{\prime}\right]\right) \\
& =\frac{2^{-v-1}}{(2 \pi)^{\frac{d}{2}}} \frac{\Gamma\left(\nu+\frac{d}{2}\right)}{\Gamma(\nu+1)} \frac{1}{\left[\cos \left(\tau-\tau^{\prime}\right)-\mathrm{e} \cdot \mathrm{e}^{\prime}\right]^{\frac{d}{2}+v}}
\end{aligned}
$$

(see also [33]). This equation expresses nothing more than the behavior of the previous Legendre's function at infinity. Not only all the $v$ 's are treated this way in one stroke but, also, one can study the boundary limit for theories corresponding to $v<-1$, even if the corresponding QFT may have no direct physical interpretation.

The two-point function of the conformal field $\widetilde{\mathcal{O}}(\eta)$ on the cone $\widehat{\mathcal{C}_{2, d}}$ corresponding to (52) is then constructed by following the prescription of Eq. (27), which yields

$$
\widetilde{W}_{v}\left(\eta, \eta^{\prime}\right)=\left(r r^{\prime}\right)^{-\frac{d}{2}-v} W_{v}^{\infty}\left([\tau, \mathrm{e}],\left[\tau^{\prime}, \mathrm{e}^{\prime}\right]\right)=\frac{1}{2 \pi^{\frac{d}{2}}} \frac{\Gamma\left(v+\frac{d}{2}\right)}{\Gamma(v+1)} \frac{1}{\left[-\left(\eta-\eta^{\prime}\right)^{2}\right]^{\frac{d}{2}+v}} .
$$

Correspondingly, we can deduce from (53) the expression of the two-point function of the associated Minkowskian field on $\mathbb{M}^{d}$, given by formula (40); by taking Eq. (24) into account, we obtain:

$$
W_{v}^{M}\left(z, z^{\prime}\right)=e^{\left(v+v^{\prime}\right)\left(\frac{d}{2}+v\right)} \widetilde{W}_{v}\left(\eta(v, z), \eta^{\prime}\left(v^{\prime}, z^{\prime}\right)\right)=\frac{1}{2 \pi^{\frac{d}{2}}} \frac{\Gamma\left(v+\frac{d}{2}\right)}{\Gamma(v+1)} \frac{1}{\left[-\left(z-z^{\prime}\right)^{2}\right]^{\frac{d}{2}+v}} .
$$


In the latter, the Poincaré coordinates $z$ and $z^{\prime}$ must be taken with the usual $i \epsilon$-prescription $\left(\operatorname{Im} z^{0}<\operatorname{Im} z^{\prime 0}\right)$, which can be checked to be implied by the spectral condition (b) of Section 2 through the previous limiting procedure.

We note that this natural way of producing the boundary field theory gives rise to the normalization advocated in [32], Eq. (2.21) (apart from a trivial factor 4, which does not depend on the anomalous dimension $\left.\Delta=\frac{d}{2}+v\right)$.

Let us now describe how the previous limiting procedure looks in the Poincaré coordinates (9). These coordinates offer the possibility of studying directly the boundary behavior of the AdS Wightman functions in a larger domain of the complex AdS spacetime. This fact is based on the following simple observation: consider the parametrization (9) for two points with complex parameters specified by

$$
\begin{aligned}
& Z=Z(v, z), \quad v \in \mathbb{R}, z \in T_{M}^{-}, \\
& Z^{\prime}=Z^{\prime}\left(v^{\prime}, z^{\prime}\right), \quad v^{\prime} \in \mathbb{R}, z^{\prime} \in T_{M}^{+} .
\end{aligned}
$$

It is easy to check that this choice of parameters implies that $Z \in T^{-}$and $Z^{\prime} \in T^{+}$. It follows that, given an AdS invariant two-point function satisfying microcausality and the normal analyticity condition $\left(b^{(i n v)}\right)$, the following restriction automatically generates a microcausal and (Poincaré) covariant two-point function on the slice $\Pi_{v}$, which satisfies the spectral condition [15] (in short: the two-point function of a general Wightman QFT):

$$
W_{\{v\}}^{M}\left(z, z^{\prime}\right)=W\left(Z(v, z), Z^{\prime}\left(v, z^{\prime}\right)\right) .
$$

On the basis of the dimensional boundary condition (18), and of the fact (obtained by comparing (5) and (9)) that $e^{v} / r=\sqrt{1+1 / r^{2}} \cos \tau+\mathrm{e}^{d}$ tends to the finite limit $\cos \tau+\mathrm{e}^{d}$ when $r$ tends to infinity, one sees that the following limit exists and that it yields (in view of (27) and (40)):

$$
\lim _{v \rightarrow+\infty} e^{2 v \Delta} W_{\{v\}}^{M}\left(z, z^{\prime}\right)=W^{M}\left(z, z^{\prime}\right) .
$$

The limiting two-point function $W^{M}\left(z, z^{\prime}\right)$ then automatically exhibits microcausality, Poincaré invariance and the spectral condition. (The invariance under special conformal transformations and scaling property would necessitate a special check, but they result from the general statement of conformal invariance of the limiting field $\widetilde{\mathcal{O}}(\eta)$ proved in Section 3 completed by the analysis of [14].)

When applied to the Wightman functions of Klein-Gordon fields (i.e., with $\Delta=d / 2$ $+v)$, the latter presentation of the limiting procedure gives immediately the result obtained in Eq. (54) but in a larger complex domain:

$$
\lim _{v \rightarrow \infty} e^{2 v\left(\frac{d}{2}+v\right)} W_{v}\left(Z(v, z), Z^{\prime}\left(v, z^{\prime}\right)\right)=\frac{1}{2 \pi^{d / 2}} \frac{\Gamma\left(v+\frac{d}{2}\right)}{\Gamma(v+1)} \frac{1}{\left[-\left(z-z^{\prime}\right)^{2}\right]^{\frac{d}{2}+v}},
$$

In a completely similar way one can compute the bulk-to-boundary correlation function by considering a two-slice restriction $W_{v}\left(Z(v, z), Z^{\prime}\left(v^{\prime}, z^{\prime}\right)\right)$ of $W_{v}$. The bulk-to-boundary correlation function is obtained by sending $v^{\prime} \rightarrow \infty$ while keeping $v$ fixed, by the following limit: 


$$
\begin{aligned}
\lim _{v^{\prime} \rightarrow \infty} e^{v^{\prime}\left(\frac{d}{2}+v\right)} W_{v}\left(Z(v, z), Z^{\prime}\left(v^{\prime}, z^{\prime}\right)\right) & =\frac{1}{2 \pi^{\frac{d}{2}}} \frac{\Gamma\left(v+\frac{d}{2}\right)}{\Gamma(v+1)} \frac{1}{\left(e^{-v}-e^{v}\left(z-z^{\prime}\right)^{2}\right)^{\frac{d}{2}+v}} \\
& =\frac{1}{2 \pi^{\frac{d}{2}}} \frac{\Gamma\left(v+\frac{d}{2}\right)}{\Gamma(v+1)}\left(\frac{u}{u^{2}-\left(z-z^{\prime}\right)^{2}}\right)^{\frac{d}{2}+v} .
\end{aligned}
$$

\section{Decomposition of AdS Klein-Gordon fields to the branes}

We will discuss in this section a decomposition of Klein-Gordon fields associated with the Poincaré coordinate system (9). This will produce some new and exact formulae which exhibit how a field of a given mass on the ambient AdS spacetime is decomposed into elementary massive fields when restricted to the brane. We will also gain insight about the two different AdS regimes depending on the values of of the mass parameter $v$. We follow here a method already used in $[34,35]$.

According to Eq. (56), we can obtain by restriction Poincaré invariant QFT's on the branes $\Pi_{v}$ of $A d S_{d+1}$. Of course the restricted theories are not conformal and can become conformal only in the limit $v \rightarrow \infty$.

Let us study the case of Klein-Gordon fields. By using the coordinates (9) the KleinGordon equation (47) is separated into the following pair of equations:

$$
\begin{aligned}
& \square_{M} \phi+\lambda \phi=0, \\
& e^{2 v}\left[\theta^{\prime \prime}(v)+d \theta^{\prime}(v)-m^{2} \theta(v)\right]=-\lambda \theta(v) .
\end{aligned}
$$

The first equation is another Klein-Gordon equation, now considered on a $d$-dimensional Minkowski spacetime. The second equation is an eigenvalue equation for a second order operator. The separation constant $\lambda$ is for the moment unrestricted. To get information on the allowed values for $\lambda$ we have to consider Eq. (61) as a spectral problem in a suitable Hilbert space. To this end let us introduce the Hilbert space $L^{2}\left(\mathbb{R}, e^{(d-2) v} \mathrm{~d} v\right)$, where the differential operator defined in Eq. (61) is symmetric. It is useful to pass to the variable $u=e^{-v}$ already introduced in Eq. (9) and define $f(u)=\theta(v) e^{\frac{d-1}{2} v}$. Eq. (61) is then turned into

$$
-f^{\prime \prime}(u)+\frac{m^{2}+\left(d^{2}-1\right) / 4}{u^{2}} f(u)=-f^{\prime \prime}(u)+\frac{(v+1 / 2)(v-1 / 2)}{u^{2}} f(u)=\lambda f(u),
$$

a well-known Schrödinger spectral problem on the half-line (the Hilbert space is now $\left.L^{2}\left(\mathbb{R}^{+}, \mathrm{d} u\right)\right)$.

Following [22, p. $88 \mathrm{ff}]$, we learn that there are two distinct regimes corresponding as before to $v \geqslant 1$ and $|v|<1$.

When $v \geqslant 1$ the previous operator is essentially self-adjoint and there is only one possible choice for the generalized eigenfunctions, namely

$$
f_{\lambda}(u)=\frac{1}{\sqrt{2}} u^{1 / 2} J_{v}(\sqrt{\lambda} u),
$$


where $J_{v}$ are Bessel's functions. The completeness of these eigenfunctions gives Hankel's formula, which expresses the resolution of the identity in $L^{2}\left(\mathbb{R}^{+}, \mathrm{d} u\right)$ as follows:

$$
g(u)=\int_{0}^{\infty} \mathrm{d} \lambda f_{\lambda}(u) \int_{0}^{\infty} f_{\lambda}\left(u^{\prime}\right) g\left(u^{\prime}\right) \mathrm{d} u^{\prime}, \quad \forall g \in L^{2}\left(\mathbb{R}^{+}, \mathrm{d} u\right) .
$$

When $0 \leqslant v<1$ both solutions $u^{1 / 2} J_{v}(\sqrt{\lambda} u)$ and $u^{1 / 2} J_{-v}(\sqrt{\lambda} u)$ are square integrable in the neighborhood of $u=0$ and must be taken into consideration: we are in the so-called limit circle case at zero [22,36], which implies that the operator is not essentially selfadjoint and there exists a $S^{1}$ ambiguity in the self-adjoint extensions we can perform. The freedom is exactly in the choice of the boundary conditions at $u=0$ (corresponding to the boundary of AdS).

Now we have a one-parameter family of eigenfunctions:

$$
f_{\lambda}^{(\varkappa)}(u) \equiv \sqrt{\frac{u}{2}}\left(\varkappa^{2}-2 \varkappa \lambda^{v} \cos (\pi v)+\lambda^{2 v}\right)^{-1 / 2}\left[\varkappa J_{v}(\sqrt{\lambda} u)-\lambda^{v} J_{-v}(\sqrt{\lambda} u)\right],
$$

to which we must add one bound state when $\varkappa>0$ :

$$
f_{\text {bound }}^{(\varkappa)}(u) \equiv \sqrt{2 \varkappa^{1 / v} \frac{\sin \pi v}{\pi v}} u^{1 / 2} K_{v}\left(\varkappa^{1 / 2 v} u\right) .
$$

The possible choices of the parameter $\varkappa$ do correspond to different self-adjoint extensions of the differential operator (62). To each such extension there is associated a domain $\mathfrak{D}^{(\varkappa)}$ also depending on the parameter $\varkappa$ [36]. To construct $\mathfrak{D}^{(\varkappa)}$ consider the one-dimensional subspaces $H_{ \pm}$spanned by the eigenfunctions solving Eq. (62) with eigenvalues $\pm i$ :

$$
f_{ \pm}(u) \equiv \sqrt{u} K_{v}\left(e^{ \pm \frac{i \pi}{4}} u\right)
$$

both these functions are square-integrable when $0 \leqslant v<1$. Each extension is in oneto-one correspondence with partial isometries $U: H_{+} \mapsto H_{-}$, namely — in this case with elements of $U(1) \simeq S^{1}$. The domain of the extension is obtained by adjoining to the original domain of symmetry the subspace $\left(\mathrm{id}_{H_{+}}+U\right) H_{+}$: here it means that we have to add the span of the $L^{2}$ element

$$
f_{\alpha}(u) \equiv f_{+}(u)+e^{i \alpha} f_{-}(u)
$$

which has in our case the asymptotics

$$
f_{\alpha}(u) \simeq \frac{\pi}{2 \sin (\pi v)}\left[\frac{2^{v}\left(e^{-i \pi v / 4}+e^{i \alpha+i \pi v / 4}\right)}{\Gamma(1-v)} u^{-v}-\frac{2^{-v}\left(e^{\mathrm{i} \pi \nu / 4}+e^{i \alpha-\mathrm{i} \pi \nu / 4}\right)}{\Gamma(1+v)} u^{\nu}\right] .
$$

The generalized eigenfunctions of the operator (62) corresponding to a specific extension have the following asymptotics

$$
f_{\lambda}^{(\varkappa)}(u) \simeq 2^{-\frac{1}{2}} u^{\frac{1}{2}}\left(\varkappa^{2}-2 \varkappa \lambda^{v} \cos (\pi v)+\lambda^{2 v}\right)^{-\frac{1}{2}} \lambda^{\frac{v}{2}}\left[\varkappa \frac{2^{-v} u^{v}}{\Gamma(1+v)}-\frac{2^{v} u^{-v}}{\Gamma(1-v)}\right] .
$$


As usual these functions do not belong to $L^{2}\left(\mathbb{R}^{+}, \mathrm{d} u\right)$ but any wave-packet does; moreover any such wave packet has this asymptotics. This allows us to find which parameter $x$ corresponds to which unitary operator $e^{i \alpha}: H_{+} \mapsto H_{-}$, i.e., to a specific self-adjoint extension. Indeed, by matching the asymptotics in Eqs. (68) with that in Eq. (69) we obtain

$$
\varkappa=\frac{\cos \left(\frac{\alpha}{2}-\frac{\pi v}{4}\right)}{\cos \left(\frac{\alpha}{2}+\frac{\pi v}{4}\right)} .
$$

We can now show that the ("bulk-to-bulk") two-point function (48) in $A d S_{d+1}$ in the whole range $v \in(-1, \infty)$ can be decomposed as follows:

$$
\begin{aligned}
& W_{v}^{d+1}\left(Z(v, z), Z^{\prime}\left(v^{\prime}, z^{\prime}\right)\right)=\int_{0}^{\infty} \mathrm{d} \lambda \theta_{\lambda}(v) \theta_{\lambda}\left(v^{\prime}\right) W_{\lambda}^{M, d}\left(z, z^{\prime}\right), \quad v \in[1, \infty), \\
& W_{v}^{d+1}\left(Z(v, z), Z^{\prime}\left(v^{\prime}, z^{\prime}\right)\right)=\int_{0}^{\infty} \mathrm{d} \lambda \theta_{\lambda}^{(\infty)}(v) \theta_{\lambda}^{(\infty)}\left(v^{\prime}\right) W_{\lambda}^{M, d}\left(z, z^{\prime}\right), \quad v \in[0,1), \\
& W_{v}^{d+1}\left(Z(v, z), Z^{\prime}\left(v^{\prime}, z^{\prime}\right)\right)=\int_{0}^{\infty} \mathrm{d} \lambda \theta_{\lambda}^{(0)}(v) \theta_{\lambda}^{(0)}\left(v^{\prime}\right) W_{\lambda}^{M, d}\left(z, z^{\prime}\right), \quad v \in(-1,0),
\end{aligned}
$$

where $W_{\lambda}^{M, d}\left(z, z^{\prime}\right)$ is the usual two-point function for a Klein-Gordon field on $\mathbb{M}^{d}$ of square mass $\lambda$ in the Wightman vacuum:

$$
\begin{aligned}
W_{\lambda}^{M, d}\left(z, z^{\prime}\right) & \equiv \int \frac{\mathrm{d}^{d} p}{(2 \pi)^{d-1}} \delta\left(p^{2}-\lambda\right) \Theta\left(p_{0}\right) e^{-i p \cdot\left(z-z^{\prime}\right)} \\
& =(2 \pi)^{-d / 2}\left(\frac{\delta}{\sqrt{\lambda}}\right)^{\frac{2-d}{2}} K_{\frac{d-2}{2}}(\sqrt{\lambda} \delta), \quad \delta \equiv-\left(z-z^{\prime}\right)^{2} .
\end{aligned}
$$

In Eqs. (70) the functions $\theta_{\lambda}^{(\infty)}$ and the $\theta_{\lambda}^{(0)}$ belong to the domains of self-adjointness corresponding to the values $\varkappa=\infty$ and $\varkappa=0$, respectively. They explicitly read

$$
\begin{aligned}
\theta_{\lambda}^{(\infty)}(v) & =\frac{1}{\sqrt{2}} e^{-\frac{d}{2} v} J_{v}\left(\sqrt{\lambda} e^{-v}\right), \\
\theta_{\lambda}^{(0)}(v) & =\frac{1}{\sqrt{2}} e^{-\frac{d}{2} v} J_{-|v|}\left(\sqrt{\lambda} e^{-v}\right) .
\end{aligned}
$$

The reason why we must use different self-adjoint extensions is that $W_{v}^{d+1}(Z(v, z)$, $Z\left(v^{\prime}, z^{\prime}\right)$ ), as a function of $v$ (or $v^{\prime}$ ) belongs to $\mathfrak{D}^{(\infty)}$ when $v \in[0,1)$ while it belongs to $\mathfrak{D}^{(0)}$ when $v \in(-1,0)$ : this can be proved directly by studying the asymptotics.

The three Eqs. (70) are thus summarized into the following formula valid for the whole range of parameter $v$ :

$$
\begin{aligned}
& W_{v}^{d+1}\left(Z(v, z), Z^{\prime}\left(v^{\prime}, z^{\prime}\right)\right) \\
& =(2 \pi)^{-\frac{d}{2}}\left(u u^{\prime}\right)^{\frac{d}{2}} \int_{0}^{\infty} \frac{\mathrm{d} \lambda}{2} \lambda^{\frac{d-2}{4}} J_{v}(\sqrt{\lambda} u) J_{v}\left(\sqrt{\lambda} u^{\prime}\right) K_{\frac{d-2}{2}}(m \delta),
\end{aligned}
$$


with, again, $u=e^{-v}$. The full details of the proof include analytical continuation to the Euclidean section where $\delta=-\left(z-z^{\prime}\right)^{2}>0$, and take into account formula (12) in [37, p. 64].

Eq. (70) can also been inverted and we obtain the Minkowski Klein-Gordon two-point function on the slice $\Pi_{v}$ by integrating $W_{v}$ against the eigenfunctions $\theta_{\lambda}$. For instance, when $v>1$ this corresponds to the introduction of the fields $\phi_{\lambda}(x)$ on the Minkowskian slice $\Pi_{v}$ obtained by smearing the AdS Klein-Gordon field $\widehat{\Phi}$ with the complete set of modes (72):

$$
\phi_{\lambda}(x)=\int_{-\infty}^{\infty} \Phi(X(v, x)) \bar{\theta}_{\lambda}(v) e^{(d-2) v} \mathrm{~d} v .
$$

It can be shown that the field $\phi_{\lambda}(x)$ is a canonical Minkowskian Klein-Gordon field in the Wightman vacuum state. In precise terms, we have that the AdS vacuum expectation value of $\phi_{\lambda}(x)$ is given by

$$
W_{\lambda, \lambda^{\prime}}\left(x, x^{\prime}\right) \equiv\left\langle\Omega\left|\phi_{\lambda}(x) \phi_{\lambda^{\prime}}\left(x^{\prime}\right)\right| \Omega\right\rangle=\delta\left(\lambda-\lambda^{\prime}\right) W_{\lambda}^{M, d}\left(z, z^{\prime}\right) .
$$

In particular, the fields $\phi_{\lambda}$ have zero correlation (and hence commute) for different values of the square mass $\lambda$.

The results of this section can be used to construct other two-point functions $W_{v}^{d+1,(\varkappa)}$ $\left(Z(v, z), Z\left(v^{\prime}, z^{\prime}\right)\right)$ for a Klein-Gordon field on AdS by using the other self-adjoint extensions: however it is not guaranteed that such $W_{v}^{d+1,(\varkappa)}$ can be extended to the other half of AdS since the definition uses the set of coordinates defined only on one half. Moreover one should prove (or disprove) the AdS invariance and analyticity properties of such states. We will not go any further in this direction in this paper.

\section{General QFT's in the Poincaré coordinates}

The results of Sections 4 and 5 suggest the following alternative approach to the $\mathrm{AdS} \rightarrow$ CFT correspondence. Starting from a given set of AdS invariant $n$-point functions satisfying general requirements of the form described in Section 2, it is (at least formally) possible to obtain a set of Poincaré invariant (see below) $n$-point functions in onedimension less by taking the following restrictions:

$$
\mathcal{W}_{n\{v\}}^{M}\left(x_{1}, \ldots, x_{n}\right)=\mathcal{W}_{n}\left(X_{1}\left(v, x_{1}\right), \ldots, X_{n}\left(v, x_{n}\right)\right) .
$$

On the basis of the requirement of asymptotic dimensionality (c) supplemented by an argument similar to the one given in Section 4.2 (based on Eqs. (27) and (40)) for justifying the limit (57) of two-point functions in the slices $\Pi_{v}, n$-point correlation functions on the boundary will be obtained by taking the following limits:

$$
\mathcal{W}_{n}^{M}\left(x_{1}, \ldots, x_{n}\right)=\lim _{v \rightarrow \infty} e^{n v \Delta} \mathcal{W}_{n\{v\}}^{M}\left(x_{1}, \ldots, x_{n}\right)
$$

One can also consider a many-leaf restriction as follows: 


$$
\begin{aligned}
& \mathcal{W}_{n\left\{v_{m+1}, \ldots, v_{n}\right\}}\left(X_{1}, \ldots, X_{m}, x_{m+1}, \ldots, x_{n}\right) \\
& \quad=\mathcal{W}_{n}\left(X_{1}, \ldots, X_{m}, X_{m+1}\left(v_{m+1}, x_{m+1}\right), \ldots, X_{n}\left(v_{n}, x_{n}\right)\right),
\end{aligned}
$$

and get various bulk-to-boundary correlation functions by taking the limit as before:

$$
\begin{aligned}
& \mathcal{W}_{n}\left(X_{1}, \ldots, X_{m}, x_{m+1}, \ldots, x_{n}\right) \\
& \quad=\lim _{v_{m+1}, \ldots, v_{n} \rightarrow \infty} e^{\left(v_{m+1}+\cdots+v_{n}\right) \Delta} \mathcal{W}_{n\left\{v_{m+1}, \ldots, v_{n}\right\}}\left(X_{1}, \ldots, X_{m}, x_{m+1}, \ldots, x_{n}\right) .
\end{aligned}
$$

Restricting ourselves here to the limiting procedure described by Eq. (78), we then see that the general AdS $\rightarrow$ CFT correspondence for QFT's described in Section 3 can alternatively be presented purely in terms of a limit of Minkowskian fields, denoted as follows:

$$
\Phi(X) \rightarrow\left\{\varphi_{v}(x)\right\} \rightarrow \mathcal{O}(x),
$$

where each field $\varphi_{v}(x)$ is the scalar Minkowskian field whose $n$-point correlation functions are those given by (77).

Here we must point out that there is a substantial difference between two-point and $n$-point functions. In fact, in view of their maximal analyticity property (see Section 4.2) the two-point functions admit restrictions to the slices $\Pi_{v}$ which are themselves boundary values of holomorphic functions in relevant Minkowskian complex domains of the corresponding complexified slices $\Pi_{v}^{(c)}$ : in this case there is therefore no problem of restriction of the distribution $\mathcal{W}_{2}$ to $\Pi_{v} \times \Pi_{v}$.

As regards the $n$-point correlation functions, the existence of the restrictions (77) as distributions on $\left(\Pi_{v}\right)^{n}$ is not an obvious consequence of the requirements (a), (b), (c) of Section 2. Only the existence of the corresponding restrictions at Euclidean points of $\left(\Pi_{v}^{(c)}\right)^{n}$ (namely the Schwinger functions of these Minkowskian theories) are direct consequences of the spectral condition (b) we have assumed: this is because changing $\tau$ into $i \sigma$ in (5) or changing $x^{0}$ into $i y^{0}$ in (9), all other parameters being kept real, yield two equivalent representations of the Euclidean points of $\widehat{A d S}_{d+1}^{(c)}$.

As a matter of fact, in order to be able to define the restrictions (77) as distributions enjoying the full structure of Minkowskian $n$-point functions, namely as distribution boundary values of holomorphic functions in relevant domains of $\left(\Pi_{v}^{(c)}\right)^{n}$, one is led to use instead of (b) an alternative spectral condition in which the positivity of the spectrum refers to the representation of a $d$-dimensional Abelian subgroup of $G$ playing the role of the Minkowskian translation group with respect to the slices $\Pi_{v}$.

Let us briefly sketch the construction. Using the horocyclic parametrization of Eq. (9), we can lift the action of the Poincare group as follows. Consider the standard action of the Poincare group on the Minkowski spacetime coordinates: $x^{\prime \mu}=\Lambda_{v}^{\mu} x^{\nu}+a^{\mu}, \mu=$ $0,1, \ldots, d-1$. By plugging this relation into Eq. (9) we promptly obtain the following relation:

$$
\left\{\begin{array}{l}
X^{\prime \mu}=\Lambda_{\nu}^{\mu} X^{v}+\left(X^{d}+X^{d+1}\right) a^{\mu}, \\
X^{\prime d}=\left(1+\frac{a^{2}}{2}\right) X^{d}+a_{\mu} \Lambda_{\nu}^{\mu} X^{\nu}+X^{d+1} \frac{a^{2}}{2} \\
X^{\prime d+1}=\left(1-\frac{a^{2}}{2}\right) X^{d+1}-a_{\mu} \Lambda_{\nu}^{\mu} X^{\nu}-X^{d} \frac{a^{2}}{2},
\end{array}\right.
$$


where Greek indices are raised and lowered with the standard Minkowski metric. In matrix form we get

$$
g(\Lambda, a)=\left(\begin{array}{ccc}
\Lambda & a & a \\
\Lambda a^{T} & \left(1+a^{2} / 2\right) & a^{2} / 2 \\
-\Lambda a^{T} & -a^{2} / 2 & \left(1-a^{2} / 2\right)
\end{array}\right) .
$$

Among such transformations there is the Abelian subgroup of Poincaré translations $g(\mathbb{I}, a)$. The corresponding generators

$$
P_{\mu} \equiv\left(X^{d}+X^{d+1}\right) \frac{\partial}{\partial X^{\mu}}+X_{\mu}\left(\frac{\partial}{\partial X^{d}}-\frac{\partial}{\partial X^{d+1}}\right)
$$

of these transformations form an Abelian algebra. The AdS spectral condition (b) of Section 2 should then be supplemented by the following one:

$\left(\mathrm{b}^{\prime}\right)$ Spectral condition: the infinitesimal generators $P^{\mu}$ are represented by (commuting) self-adjoint operators whose joint spectrum is contained in the forward light-cone $V^{+}=$ $\left\{p^{\mu} p_{\mu} \geqslant 0, p^{0} \geqslant 0\right\}$ of a d-dimensional Minkowski momentum space.

By using a Laplace transform argument $[15,36]$, in the corresponding vector variables $x_{1}, \ldots, x_{n}$ one can see that this spectral condition implies the following analyticity property of the Wightman functions:

Analyticity corresponding to the spectrum of Poincaré translations: each AdS distribution $\mathcal{W}_{n}\left(X_{1}\left(v_{1}, x_{1}\right), \ldots, X_{n}\left(v_{n}, x_{n}\right)\right)$ is the boundary value of a holomorphic function $W_{n}\left(Z_{1}\left(v_{1}, z_{1}\right), \ldots, Z_{n}\left(v_{n}, z_{n}\right)\right)$ which is defined in the tube

$$
\begin{aligned}
\mathcal{T}_{n}=\{ & Z=\left(Z_{1}, \ldots, Z_{n}\right) \in A d S_{d+1}^{(c)} ; Z_{j}=Z_{j}\left(v_{j}, z_{j}\right) ; v_{1}, \ldots, v_{n} \in \mathbb{R}, \\
& \left.\operatorname{Im}\left(z_{j+1}-z_{j}\right) \in V^{+}, j=1, \ldots, n-1\right\} .
\end{aligned}
$$

Property $\left(b^{\prime}\right)$ implies in particular that it is meaningful to consider the restricted distributions $\mathcal{W}_{n\{v\}}^{M}$ given in Eq. (77). The Poincaré invariance of $\mathcal{W}_{n\{v\}}^{M}$ follows immediately by Eq. (82). Furthermore, the positive-definiteness of this family of distributions is induced as before by the analogous property satisfied by the distributions $\mathcal{W}_{n}$ on $\widehat{A d S}_{d+1}$. We also note that the validity of the Euclidean cluster property for $W_{n\{v\}}^{M}$ is equivalent to the condition introduced earlier in Eq. (19). Under these conditions the reconstruction procedure is now justified and the given field on $\widehat{A d S}_{d+1}$ yields by restriction a well-defined quantum field $\varphi_{v}(x)$.

Moreover, it follows from the microcausality postulate (a) together with Eqs. (12) that the field $\varphi_{v}$ also satisfies standard local commutativity in $\Pi_{v}$. Finally, in view of $\left(\mathrm{b}^{\prime}\right)$, the $n$-point functions of $\varphi_{v}$ are (for each $v$ ) boundary values of holomorphic functions in the tube domains $T_{n}^{M}$ of Wightman's QFT. This shows that these theories satisfy a standard energy-momentum spectrum condition (with respect to the generators of spacetime translations). The conformal covariance of the boundary field $\mathcal{O}(x)$ results from the general analysis of Section 3.

The interesting question whether the spectral condition $\left(b^{\prime}\right)$ might be derived from condition (b) together with AdS invariance will be left for future work. 


\section{References}

[1] J. Maldacena, Adv. Theor. Math. Phys. 2 (1998) 231, hep-th/9711200.

[2] S.S. Gubser, I.R. Klebanov, A.M. Polyakov, Phys. Lett. B 428 (1998) 105, hep-th/9802109.

[3] E. Witten, Adv. Theor. Math. Phys. 2 (1998) 253, hep-th/9802150.

[4] O. Aharony, S.S. Gubser, J. Maldacena, H. Ooguri, Y. Oz, hep-th/9905111.

[5] V. Balasubramanian, P. Kraus, A. Lawrence, Phys. Rev. D 59 (1999) 046003, hep-th/9805171.

[6] S.J. Avis, C.J. Isham, D. Storey, Phys. Rev. D 18 (1978) 3565.

[7] C. Fronsdal, Phys. Rev. D 10 (1974) 589.

[8] P.A.M. Dirac, Ann. Math. 36 (1935) 657.

[9] K.-H. Rehren, hep-th/9905179.

[10] R. Haag, Local Quantum Physics, Springer-Verlag, Berlin, 1992.

[11] E. D'Hoker, D.Z. Freedman, Nucl. Phys. B 544 (1999) 612, hep-th/9809179.

[12] E. D'Hoker, D.Z. Freedman, S.D. Mathur, A. Matusis, L. Rastelli, Nucl. Phys. B 562 (1999) 353.

[13] E. D’Hoker, J. Edrmenger, D.Z. Freedman, M. Perez-Victoria, hep-th/0003218.

[14] M. Luscher, G. Mack, Commun. Math. Phys. 41 (1975) 203.

[15] R.F. Streater, A.S. Wightman, PCT, Spin and Statistics, and All That, Benjamin, 1964.

[16] T. Banks, M.R. Douglas, G.T. Horowitz, E. Martinec, hep-th/9808016.

[17] J. Bros H. Epstein, U. Moschella, in preparation.

[18] J. Bros, U. Moschella, J.P. Gazeau, Phys. Rev. Lett. 73 (1994) 1746.

[19] J. Bros, U. Moschella, Rev. Math. Phys. 8 (1996) 327, gr-qc/9511019.

[20] L. Randall, R. Sundrum, Phys. Rev. Lett. 83 (1999) 3370, hep-ph/9905221.

[21] P. Breitenlohner, D.Z. Freedman, Ann. Phys. 144 (1982) 249.

[22] E.C. Titchmarsh, Eigenfunction Expansion Associated with Second Order Differential Equations, Clarendon Press, 1962.

[23] J. Bros, G.A. Viano, Forum Math. 8 (1996) 659-722.

[24] J. Faraut, in: Lect. Notes in Math., Vol. 497, Springer-Verlag, Berlin, 1975.

[25] N.Ja. Vilenkin, Fonctions Spéciales et Théorie de la Représentation des Groupes, Dunod, Paris, 1969.

[26] B. O'Neill, Semi-Riemannian Geometry, Academic Press, 1983.

[27] S.W. Hawking, Commun. Math. Phys. 43 (1975) 199.

[28] G.W. Gibbons, S.W. Hawking, Phys. Rev. D 15 (1977) 2738.

[29] G.L. Sewell, Ann. Phys. 141 (1982) 201.

[30] G. Mack, I.T. Todorov, Phys. Rev. D 8 (1973) 1764.

[31] H. Bateman, Higher Transcendental Functions, McGraw-Hill, 1954.

[32] I.R. Klebanov, E. Witten, hep-th/9905104.

[33] S.B. Giddings, hep-th/9907129.

[34] M. Bertola, V. Gorini, U. Moschella, R. Schaeffer, Phys. Lett. B 462 (1999) 249, hepth/9906035.

[35] M. Bertola, J. Bros, V. Gorini, U. Moschella, R. Schaeffer, hep-th/0003098.

[36] M. Reed, B. Simon, Fourier analysis and self-adjointness, Methods of modern mathematical physics, Vol. 2, Academic Press, 1975.

[37] H. Bateman, Tables of Integral Transforms, McGraw-Hill, 1954. 